\title{
VERSITA
}

\section{Acta Geophysica}

vol. 56, no. 3, pp. 669-697

DOI: $10.2478 / \mathrm{s} 11600-008-0031-0$

\section{Double-averaged velocity profiles over fixed dune shapes}

\author{
Stephen R. MCLEAN ${ }^{1}$, Vladimir I. NIKORA ${ }^{2}$, \\ and Stephen E. COLEMAN ${ }^{3}$ \\ ${ }^{1}$ Mechanical Engineering Department, University of California, \\ Santa Barbara, CA, USA \\ e-mail: mclean@engineering.ucsb.edu (corresponding author) \\ ${ }^{2}$ School of Engineering, University of Aberdeen, \\ Aberdeen, United Kingdom; \\ e-mail: v.nikora@abdn.ac.uk \\ ${ }^{3}$ Department of Civil and Environmental Engineering, University of Auckland, \\ Auckland, New Zealand; \\ e-mail: s.coleman@auckland.ac.nz
}

\begin{abstract}
Spatially averaged profiles of time averaged velocity, using integrals over thin horizontal slabs (Cartesian double average), are employed in characterizing the flow over fixed dune shapes. For comparison the spatial averaging method of Smith and McLean (1977) that averages along lines at constant distance from the local bed elevation is also investigated. The Cartesian double averaged profiles of the inverse of the velocity shear are nearly constant below the crest elevation, but increase rapidly above the crest level. This results in velocity profiles that increase linearly with distance from the bed below the crest. Above the crest it can be argued that the velocity increases logarithmically, but a power law profile can also be argued. Spatially averaged eddy viscosity profiles are calculated by multiplying the average Reynolds stress by the inverse shear. The resulting profile is more complex than the uniform flow counterpart.
\end{abstract}

Key words: boundary layers, double-average, dune, law of the wall, mixing, separation, spatial averaging, velocity profile.

(c) 2008 Institute of Geophysics, Polish Academy of Sciences 


\section{INTRODUCTION}

Flow over rough boundaries such as that over ripples and dunes is highly complex and defies simple analysis. There is significant spatial variability in both the streamwise and vertical directions, typically including upstream flow due to separation. Also, due to the separation process, much of the flow is significantly more turbulent than uniform turbulent boundary layers (i.e., $\sigma_{\text {sep }}^{2}=\overline{u_{i}^{\prime} u_{i}^{\prime}} \gg \sigma_{\text {flat }}^{2}$, where $u_{i}$ is the instantaneous velocity component in the $i$ th direction, the over bar denotes Reynolds averaging, and primes denote fluctuating velocity component). These factors conspire against simplistic characterization of the flow. Nevertheless spatial averaging (averaging over horizontal scales of the size of the bottom features) has proved to be useful in characterizing the general flow properties and aiding in prediction and analysis of rough-bed flows (Raupach et al. 1991, Gimenez-Curto and Corniero Lera 1996, Nikora et al. 2001, 2007a).

Just as Reynolds averaging quantifies the effects of unsteadiness such as turbulent fluxes and generally eliminates time variations, spatial averaging eliminates the variations in space and quantifies fluxes associated with correlations in space. There is no unique spatial averaging technique, but virtually all average in time as well as space, thus they are double averaging techniques. Some average over boundary fitting coordinates and some average in a Cartesian framework. Some might average along non-orthogonal coordinates that stretch and shrink to compensate for changing depth, others might average along orthogonal, curvilinear coordinates. Here we will address two different spatial averaging schemes, comparing and contrasting them. We will utilize data from our own research as well as that of other investigators. A summary of the experiments that are used here can be found in Table 1.

\section{BOUNDARY-FITTED DOUBLE AVERAGED VELOCITY}

One of the first attempts to use spatial averaging for flow over dunes was done by Smith and McLean (1977) (hereafter referred to as S\&M). They used measurements from a 2-meter tall frame that was lowered to the bed of the Columbia River in a field of dunes 70-100 $\mathrm{m}$ long and 2-3 $\mathrm{m}$ high. The frame was repeatedly lowered to different locations over a couple of dune wavelengths. The frame held singlets (near the bed) and triplets of directional current meters, spaced approximately logarithmically from the bed. These provided mean velocity near the bed and mean velocity and estimates of the Reynolds stress further from the bed. However, it was not possible to determine accurately the frame position relative to the dunes. Also there was large variability introduced by the presence of smaller 'megaripples' and ripples on the dunes. These two factors contributed to a very noisy data set in which it was very difficult to discern the spatial patterns. Furthermore, it was impossible to obtain meaningful information about the actual direction of the 
mean flow relative to the plane defined by the mean bed elevation, therefore the data was analyzed with respect to the local streamline direction. Because the surface of the bedforms themselves were streamlines and because the frame either sat on the bed or was suspended a specific distance above the local bed elevation $\eta(x, y, t)$ each profile was measured at fixed distance from the local bed. Therefore it was logical to spatially average by averaging data collected at the same distance from the bed.

Separated flow over a dune is dominated by the turbulence that is generated by the shear layer that forms above the separation region above the trough. Turbulence levels $\left(\sigma^{2}\right)$ can be an order of magnitude greater than

Table 1

Characteristics of laboratory measurements: $\lambda$ and $\Delta$ are the length and height of the dune, $h$ is the mean water depth, $\left(u_{*}\right)_{T}$ is the shear velocity associated with the total boundary shear stress and $U$ is the mean velocity

\begin{tabular}{|c|c|c|c|c|c|c|c|}
\hline Run & Symbol & $\begin{array}{c}\lambda \\
{[\mathrm{mm}]}\end{array}$ & $\begin{array}{c}\Delta \\
{[\mathrm{mm}]}\end{array}$ & $\lambda / \Delta$ & $\begin{array}{c}h \\
{[\mathrm{~mm}]}\end{array}$ & $\begin{array}{l}\left(u_{*}\right)_{T} \\
{[\mathrm{~m} / \mathrm{s}]}\end{array}$ & $\begin{array}{c}U \\
{[\mathrm{~m} / \mathrm{s}]}\end{array}$ \\
\hline MNW $2^{1)}$ & + & 810 & 40 & 20 & 158 & 0.0327 & 0.39 \\
\hline MNW $3^{1)}$ & $\mathbf{x}$ & 810 & 40 & 20 & 546 & 0.0200 & 0.28 \\
\hline MNW $4^{1)}$ & $\Delta$ & 408 & 40 & 10 & 159 & 0.0371 & 0.38 \\
\hline MNW $5^{1)}$ & 4 & 408 & 40 & 10 & 159 & 0.0221 & 0.20 \\
\hline MNW $6^{1)}$ & $\nabla$ & 408 & 40 & 10 & 300 & 0.0458 & 0.54 \\
\hline MNW $7^{1)}$ & 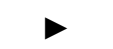 & 408 & 40 & 10 & 560 & 0.0190 & 0.24 \\
\hline $\mathrm{C} 9^{2)}$ & $\nabla$ & 750 & 40 & 19 & 120 & 0.0424 & 0.50 \\
\hline C $10^{2)}$ & $<$ & 750 & 40 & 19 & 150 & 0.0424 & 0.55 \\
\hline $\mathrm{C} 11^{2)}$ & $D$ & 750 & 40 & 19 & 180 & 0.0412 & 0.58 \\
\hline $\mathrm{C} 12^{2)}$ & $\triangle$ & 750 & 40 & 19 & 240 & 0.0387 & 0.59 \\
\hline $\mathrm{VM} T 5^{3)}$ & * & 1600 & 80 & 20 & 252 & 0.0469 & 0.39 \\
\hline VM T6 $6^{3)}$ & * & 1600 & 80 & 20 & 334 & 0.0527 & 0.51 \\
\hline $\mathrm{LAD}^{4)}$ & ० & 660 & 30 & 22 & 200 & 0.0291 & 0.21 \\
\hline $\mathrm{Ma} \mathrm{T} 2^{5)}$ & $x$ & 800 & $\left.80^{*}\right)$ & $13-40$ & 173 & 0.0414 & 0.36 \\
\hline $\mathrm{Ma} \mathrm{T} 3^{5)}$ & צ'צ' & 800 & $\left.80^{*}\right)$ & $13-40$ & 561 & 0.0214 & 0.26 \\
\hline
\end{tabular}

See references: ${ }^{1)}$ McLean et al. (1994), ${ }^{2)}$ Coleman et al. $(2006),{ }^{3)}$ van Mierlo and de Ruiter (1988), ${ }^{4)}$ Best and Kostaschuk (2002), and ${ }^{5)}$ Maddux et al. (2003a, b).

${ }^{*}$ Difference in height between highest crest and lowest trough; average height was $40 \mathrm{~mm}$. 
what one would expect in a uniform turbulent boundary layer. However, beneath this highly turbulent wake flow and downstream of reattachment, an internal boundary layer develops. Because it is over the upward-sloping stoss region of the dune that sediment is actively transported, S\&M chose to average only over this part of the dune. The resulting spatially averaged velocity $\left(u_{m}\right)$ profiles varied somewhat logarithmically, but when plotted semilogarithmically, they exhibited a concave shape rather than a perfectly straight line (see Fig. 1). The slope of the velocity profiles (plotted semilogarithmically) increased with distance from the boundary indicating that the shear velocity increased. S\&M argued that by averaging only over the stoss region of the dune, the slope of the near-bed spatially averaged velocity should reflect the shear velocity associated with the skin friction (boundary shear stress associated with grain roughness) and in the outer part of the flow the slope would reflect the skin friction plus the form drag associated with bedforms

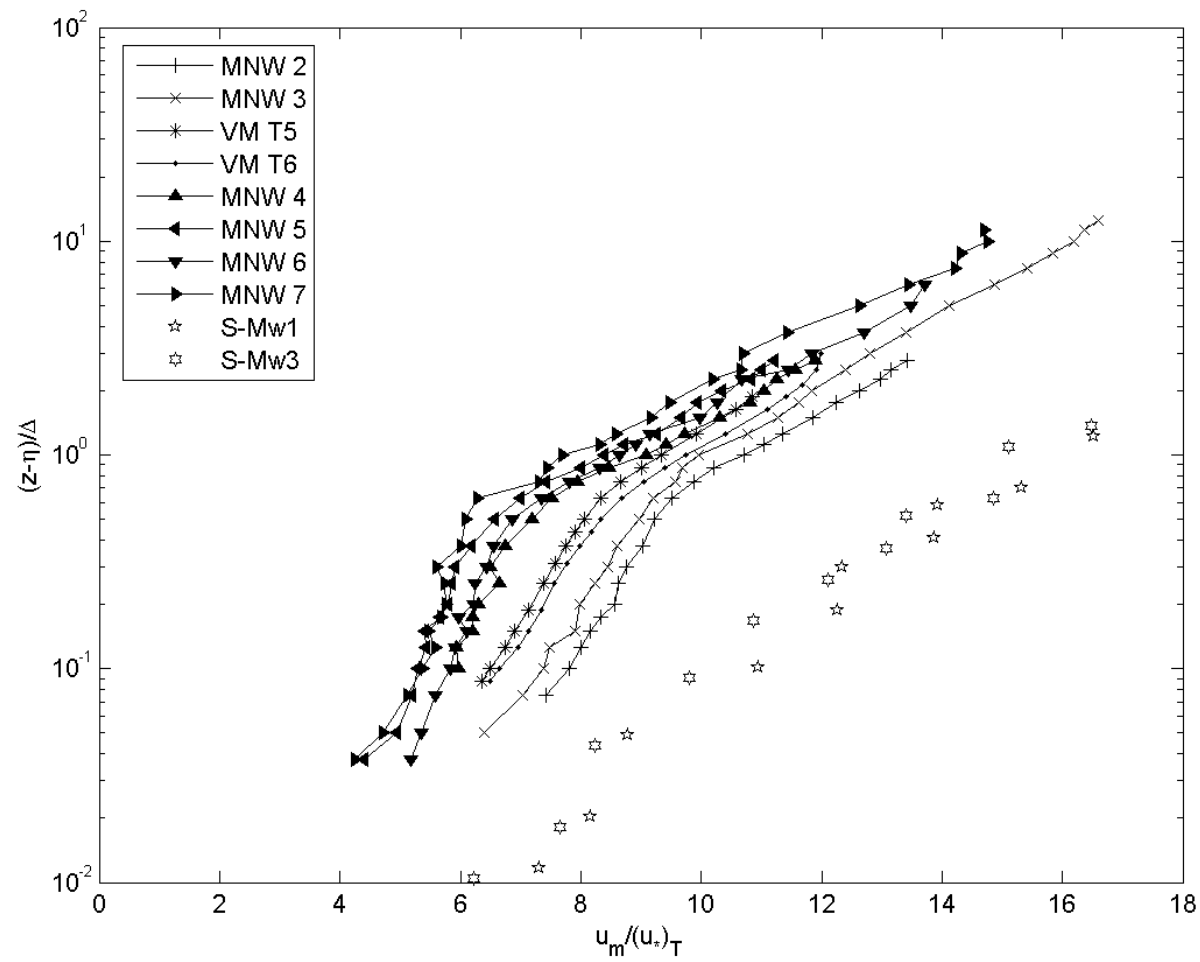

Fig. 1. Velocity profiles resulting from spatial averaging at constant distance from the local bed elevation, from reattachment to the crest of the dunes. The five- and six-pointed stars are the S\&M data from the Columbia River where $\lambda / \Delta \approx 27$; the solid triangles are from laboratory measurements with $\lambda / \Delta=10$ and the other symbols are lab measurements where $\lambda / \Delta=20$, respectively. Velocity is non-dimensionalized by the shear velocity associated with the estimated total boundary shear stress; the distance from the bed is non-dimesionalized by the height of the dunes. 
that were present. For a simple boundary where there was only one scale of bedforms this would yield a logarithmic profile with a kink in it. In the case of the Columbia River dunes, there were large megaripples having lengths of the order of several meters and heights up to a half meter propagating on the backs of the larger dunes and for lower flows there were likely small ripples 'riding' on top of the megaripples as well, therefore the arguments of S\&M would suggest several kinks in the log profile yielding a gradual concavity as is pictured in Fig. 1.

Since the data of S\&M were collected, there have been a number of careful experiments (e.g., McLean et al. 1994, Coleman et al. 2006, van Mierlo and de Ruiter 1988) conducted in the laboratory investigating flow over fixed beds having dune-like shapes. The details of these experiments are included in Table 1. In this setting and with instruments such as laser-Doppler velocimeters and Acoustic-Doppler velocimeters, it has been possible to gather data of much higher quality than was possible in the field thirty years ago. A sample of observations from the laboratory, spatially averaged over the stoss region of the dune shapes (from reattachment to the crest to be exact) is presented in Fig. 1 along with the S\&M field data. Here, where there is only a single scale of bedform, we see that the kink is highly visible, somewhat supporting the postulate of S\&M; however, there is a great deal of variability in these profiles even though they are plotted non-dimensionally to minimize variation. In the case of the S\&M study there was no way to measure the form drag or the total boundary shear stress; it was inferred from the analysis of flow and Reynolds stress measurements. In the laboratory cases the total boundary shear stresses were inferred from spatial averages of the Reynolds stress (McLean and Nikora 2006) and are known with a reasonable degree of accuracy. The fact that the field data differ significantly from the laboratory data suggests that the total shear stress for the field data was significantly underestimated.

S\&M provide equations that predict the spatially averaged velocity profile. They suggest that given a reasonable estimate of the roughness parameter $z_{0}$ and given a single input such as the spatially averaged velocity at a reference point, their equations not only predict the velocity profile but also the skin friction and the total boundary shear stress. This prediction is compared with the laboratory data in Fig. 2 (solid line). This passes through the data points but the slope of the line is clearly much smaller than that of the data; thus the value of $\left(u_{*}\right)_{T}=\sqrt{\tau_{T} / \rho}$ severely underestimates the total boundary shear stress $\tau_{T}$. If the total boundary shear stress is imposed, then the prediction significantly overpredicts the velocity (dot-dash line). If the total boundary shear stress is imposed and a reference velocity is imposed, letting $z_{0}$ serve as a free parameter, the dotted line results. Obviously this extra degree of freedom improves the comparison, but the slope of the profile is clearly in error in the near bed part of the profile. One can adjust the parame- 
ters (the drag coefficient and the coefficient that predicts the matching level) that S\&M suggest and get a significantly better comparison; however, the purpose of this paper is rather to investigate the double averaged velocity field.

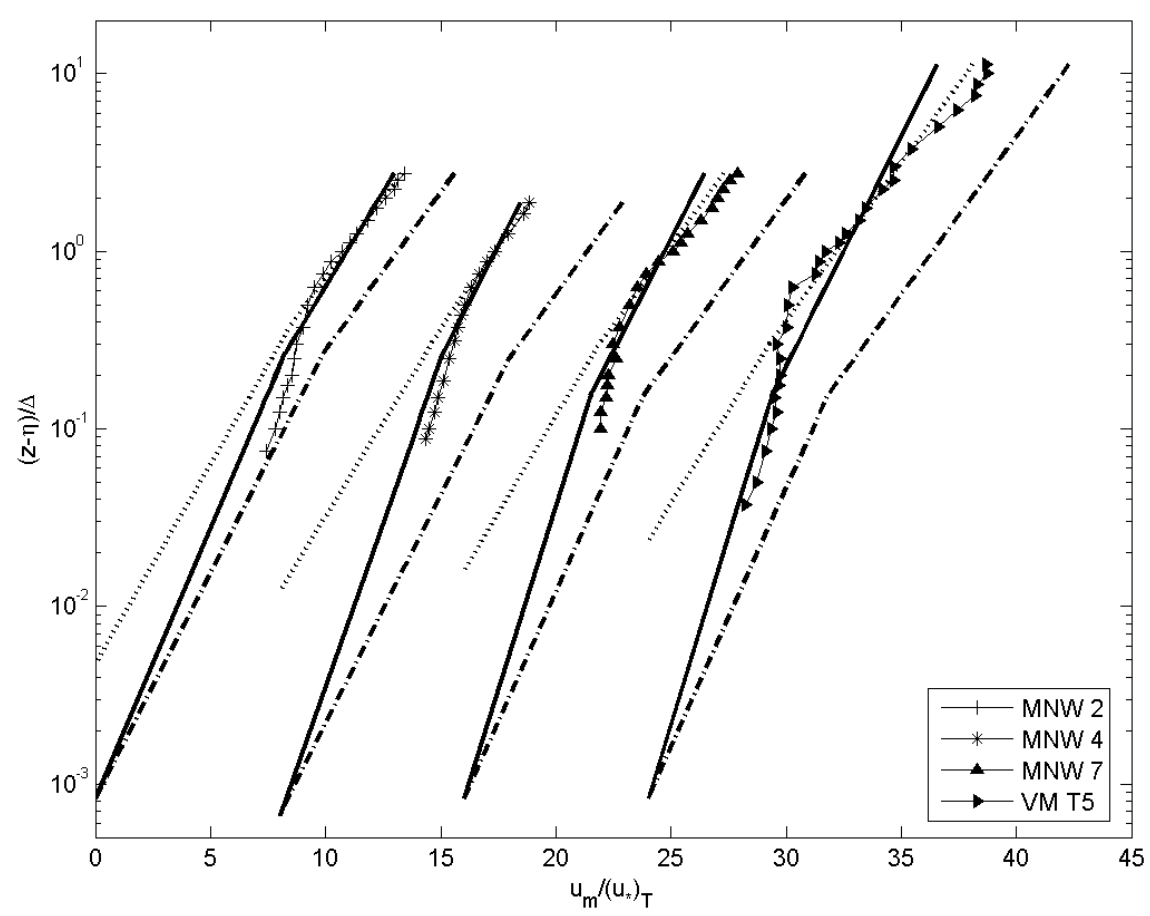

Fig. 2. Spatially averaged velocity $\left(u_{m}\right)$ profiles (shifted horizontally for clarity) with predictions from S\&M: solid line: roughness parameter for the inner part of the profile is $z_{0}$ specified by $d_{50} / 30$, and the shear velocity associated with the total stress is estimated from the measured velocity using $\left(u_{*}\right)_{T}=\kappa u_{r} / \log \left[z_{r} /\left(z_{0}\right)_{T}\right]$ where $\left(z_{0}\right)_{T}$ is the roughness parameter for the outer part of the profile; dot-dash line: $z_{0}$ specified by $d_{50} / 30$ and $\left(u_{*}\right)_{T}$ from Table 1 or from S\&M (for filed data); dotted line: $\left(u_{*}\right)_{T}$ specified from Table 1 or S\&M and $z_{0}$ selected so $u\left(z_{r}\right)=u_{r}$ the reference velocity.

This averaging approach is somewhat intuitive and it provides insight into the nature of flow over dunes; however, it is not linked to the equations of motion in a mathematically rigorous way, nor does it provide a framework for investigating mass or momentum balances. More recently rigorous techniques for averaging the equations of motion have been developed. Such techniques isolate the physical processes and provide a framework for analysis.

\section{CARTESIAN DOUBLE-AVERAGED VELOCITY}

Raupach et al. (1991), Gimenez-Curto and Corniero Lera (1996), Finnigan (2000) and Nikora et al. (2001, 2007a, b) set out in detail the concept of 
double averaging of the equations of motion in order to investigate the characteristics of complex flows such as those occurring over rough beds like those covered by dunes. This methodology not only averages in time as the Reynolds equations do, but it also averages over space. In rough-bed-flows a convenient spatial average is made by integrating over a volume that has the form of a thin horizontal slab whose horizontal extent is sufficient to encompass the features of interest. In the case of dunes this is done by averaging data over horizontal planes parallel to the mean bed elevation. For a natural dune field this would of necessity include a number of dunes because of variability in dune geometry. In two-dimensional laboratory experiments where dune shapes are identical, averaging time-averaged quantities over a single wavelength provides an accurate double average. Above the highest crest such averages are quite simple; below the crests the averaging volume is only partially filled with fluid and partially filled with solid, therefore lateral extrapolation between measurement points and the boundary must be made, which can be a significant source of error. However, knowing that near-bed velocity tends to vary logarithmically with distance from the bed, such uncertainty can be minimized.
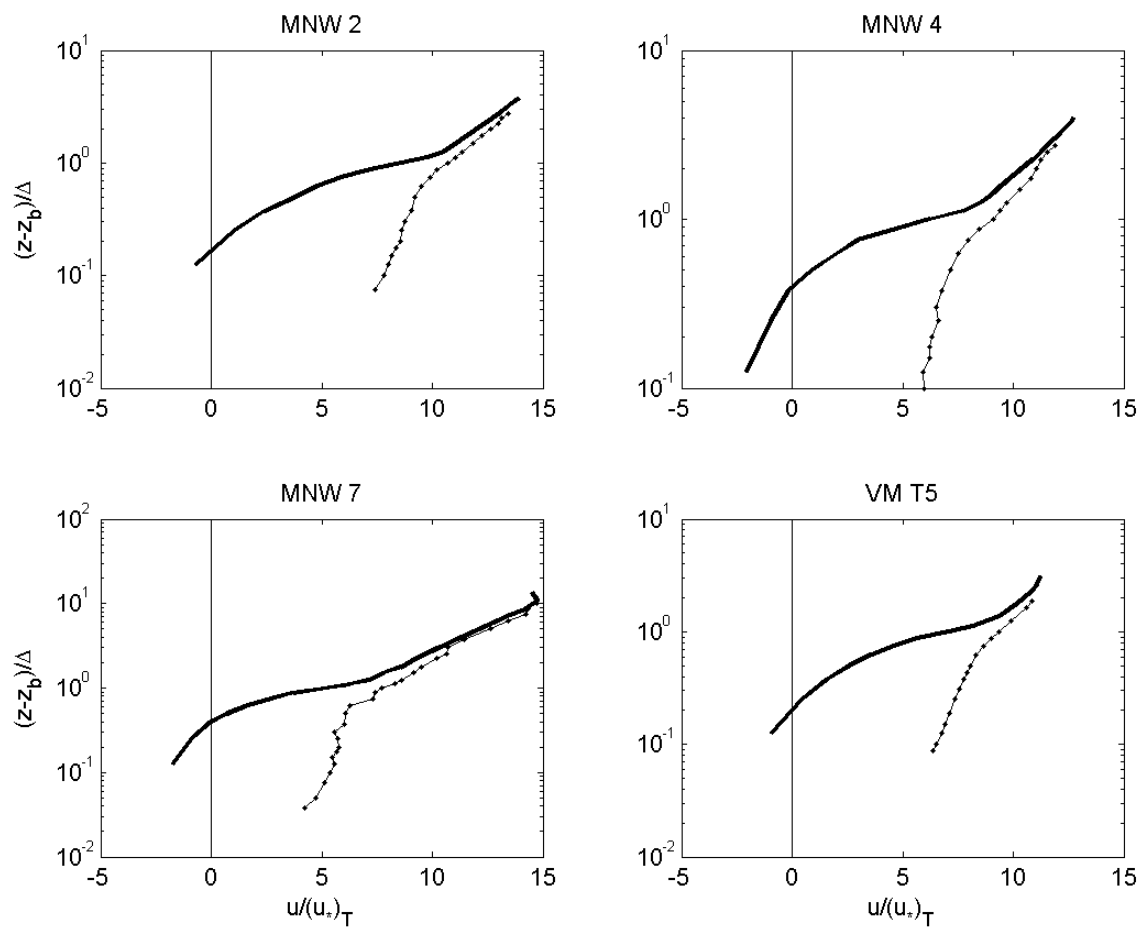

Fig. 3. Non-dimensional double-averaged velocity profiles (solid lines) and spatially-averaged velocity profiles (points). The double-averaged profiles are plotted relative to the trough elevation $\left(z_{b}=z_{t}\right)$; the spatially-averaged profiles are relative to the local bed elevation $\left(z_{b}=\eta\right)$. 
Sample double-averaged velocity profiles are plotted in Fig. 3 along with spatial averages as was done by S\&M. It should be noted that this is a bit like comparing apples to oranges but nevertheless it is interesting to compare. It should also be noted that the origin of the double averaged profiles is in the trough whereas the origin of the S\&M spatial average is the local bed elevation. The most striking difference in these profiles is the near-bed velocity. Because the double averaging method integrates over all of the flow, it includes the separation region and thus yields negative velocities near the trough elevation. Further from the bed the two profiles tend to merge toward one another but the shapes are markedly different. At this point it is good to mention that in investigating logarithmic profiles the determination of the appropriate origin is of paramount importance. The shape and slope of the profiles is profoundly affected by changes in the origin.

\section{Averaged velocity above crest level}

The double-averaged profiles in Fig. 3 exhibit a quasi-logarithmic region in the outer flow but, unlike the spatially-averaged S\&M profiles, the inner part of the flow seems to vary linearly with distance from the bed as has been reported in a number of studies (Nikora et al. 2001, 2004, Nikora and McLean 2001, Coleman et al. 2006, 2007, McLean and Nikora 2006). This linear region is discussed in more detail below, but first the logarithmic part of the profile will be addressed.

As stated above, one of the most challenging aspects of investigating turbulent flows over rough boundaries is determining the appropriate origin for the vertical coordinate. Ordinarily a natural datum for the vertical coordinate manifests itself. This could be the crest elevation or the trough elevation for regular dune shapes, or it could be the highest crest or lowest trough for a natural dune field. However, the flow itself 'sees' a natural origin that differs by the so-called 'displacement thickness' from the datum and this must be determined. For uniform (flat bed) turbulent flows the flow varies logarithmically, thus the shear is inversely proportional to the elevation above the bed (law of the wall):

$$
\frac{\partial u}{\partial z}=\frac{u_{*}}{\kappa z},
$$

where $u$ is the local (double averaged) streamwise velocity, $z$ is the vertical coordinate (elevation above the flat bed), $u_{*}=\sqrt{\tau_{b} / \rho}$ is the shear velocity, $\tau_{b}$ is the boundary shear stress, $\rho$ is the fluid density and $\kappa \approx 0.4$ is von Karman's constant. Nikora et al. (2002) suggested using this assumption for determining the displacement thickness $d_{0}$ for rough-bed flows. Rearranging (1):

$$
\frac{1}{\partial u / \partial z}=\frac{\kappa}{u_{*}}\left(z-d_{0}\right)
$$


Thus the intercept $d_{0}$ of a linear regression to the inverse shear plotted versus an arbitrary datum will yield the displacement of the origin from that datum and the slope will be inversely proportional to the shear velocity. In this paper we assume that $u_{*}=\left(u_{*}\right)_{T}=\sqrt{\tau_{T} / \rho}$ where $\tau_{T}$ is the total stress including form drag and skin friction. The non-dimensional inverse shear data are plotted in Fig. 4. The linear regression to all the data also appears as the solid line in this figure along with the values of $\kappa$ and $d_{0, \text { lin }}$ for the regression. Two observations arise from this plot: (1) the inverse shear is not very linear over this range and (2) there appears to be a difference between the runs for which $\lambda / \Delta=20$ and $\lambda / \Delta=10$, where $\lambda$ and $\Delta$ are the wavelength and height of the dunes, respectively. In the linear regression shown here the slope of the line is dominated by the outer part of the flow and $\kappa=0.285$ is less than the commonly held value of von Karman's constant (0.4).

According to Barenblatt (2003) dimensional analysis tells us that the velocity shear is

$$
\frac{\partial u}{\partial z}=\frac{u_{*}}{z} f\left(\frac{z}{\delta}, R e\right)
$$

where $\delta=v / u_{*}$ is the viscous length scale, $v$ is the kinematic viscosity and $R e=\bar{u} h / v$ is the Reynolds number based on the depth-averaged velocity and the water depth. Assuming a specific form for the function $f$ and rearranging yields:

$$
\frac{u_{*} / \Delta}{\partial u / \partial z}=f_{2}\left(\frac{z-d_{0}}{\Delta}\right)^{\alpha(R e)},
$$

where $f_{2}, d_{0}$ and $\alpha$ are constants to be determined by a least squares fit to the inverse shear. If $\alpha=1$ a logarithmic velocity profile (with origin at $d_{0}$ ) will result, but the non-linear appearance of the inverse shear in Fig. 4 suggests that the velocity might be better described by a power law. Because of the form of the equation, (4) does not lend itself directly to a least square fit; however, if a value of $d_{0}$ is chosen, a linear regression of the log of both sides of (4) yields the other two constants $f_{2}$ and $\alpha$. The value of $d_{0}$ can then be varied until the correlation coefficient is maximized. The resulting fit also appears in Fig. 4 (dashed line), along with the values of $\alpha$ and $d_{0, p o w}$. Integrating (4) the velocity becomes

$$
\frac{u}{u_{*}}=\frac{1}{f_{2}(1-\alpha)}\left(\frac{z-d_{0}}{\Delta}\right)^{1-\alpha}+f_{1},
$$

where $f_{1}$ is a constant of integration. This power-law fit clearly matches the inverse shear data in Fig. 4 better than the simple linear fit suggesting that the outer part of the double-averaged velocity profile is not logarithmic. However, before abandoning the idea of logarithmic profiles altogether, it 


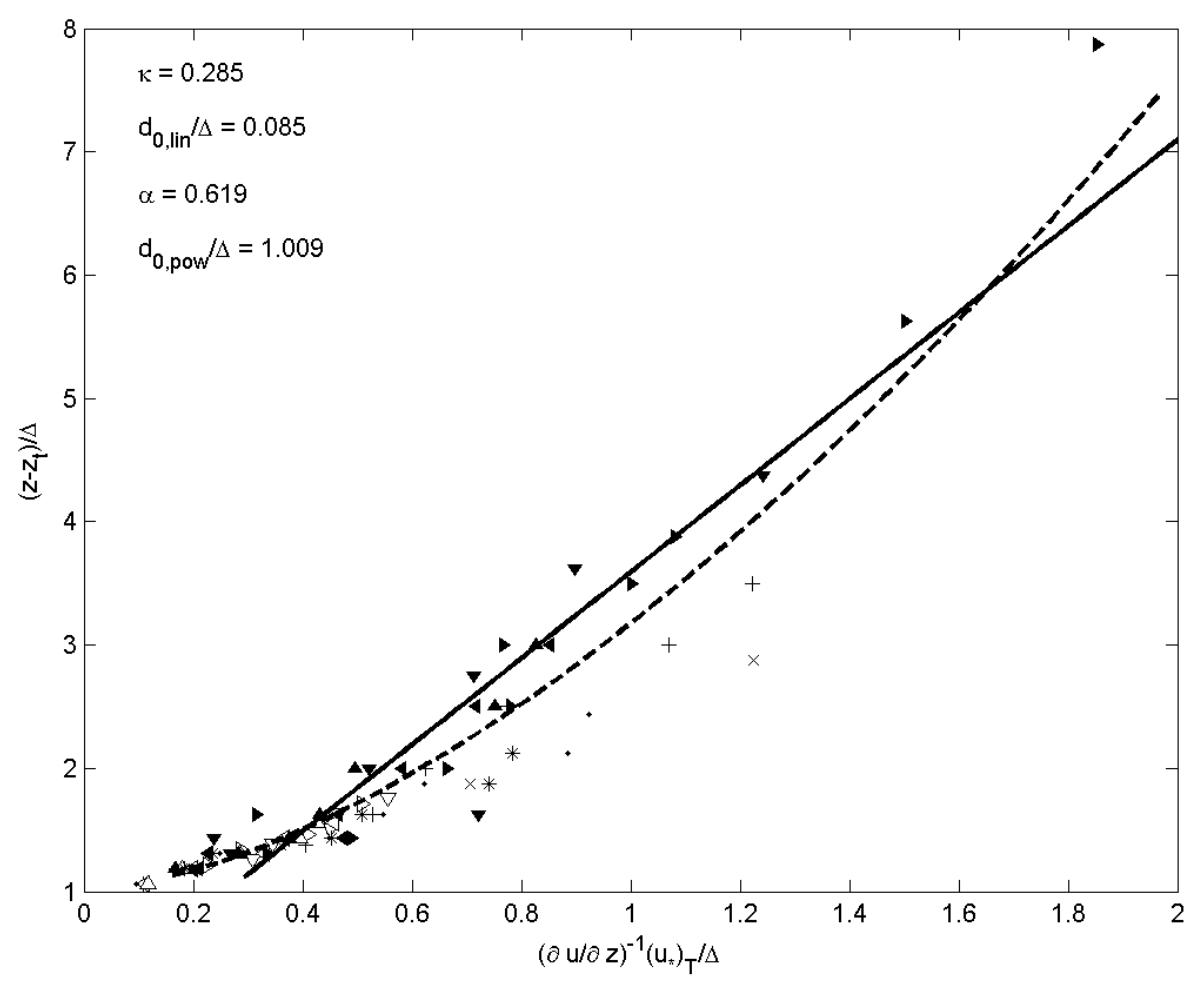

Fig. 4. Non-dimensional double-averaged inverse velocity shear plotted versus nondimensional elevation above the trough. Only data above the crest elevation are included. The solid line is a linear regression to the data; the dashed line is a fit of (4) to the data (see Table 1 for symbol definitions).

should be noted that in the region just above the crest, with the exception of a couple of outlying points, virtually all the inverse shear data lie along a straight line, for all aspect ratios $\lambda / \Delta$. Furthermore, in the outer part of the profiles there is also some suggestion that the inverse shear varies linearly. In Fig. 5 intersecting straight lines are plotted for comparison with the measured data. In comparing Figs. 4 and 5 it is difficult to choose which is better in representing the inverse shear data. Perhaps comparing with the velocity can add some insight.

Unlike the inverse shear where data from the different laboratory runs can be combined to yield a universal (though somewhat noisy) profile, the constants that arise in integrating the shear to find velocity cause profiles to vary significantly from run to run. Basically the constants of integration represent effective bottom roughness, which appears to be dependent on more than just the aspect ratio of the dune. This is readily seen in Fig. 6 where the non-dimensional velocity profiles (for double-averaged data above the crest level) are plotted. In spite of non-dimensionalizing by the shear ve- 


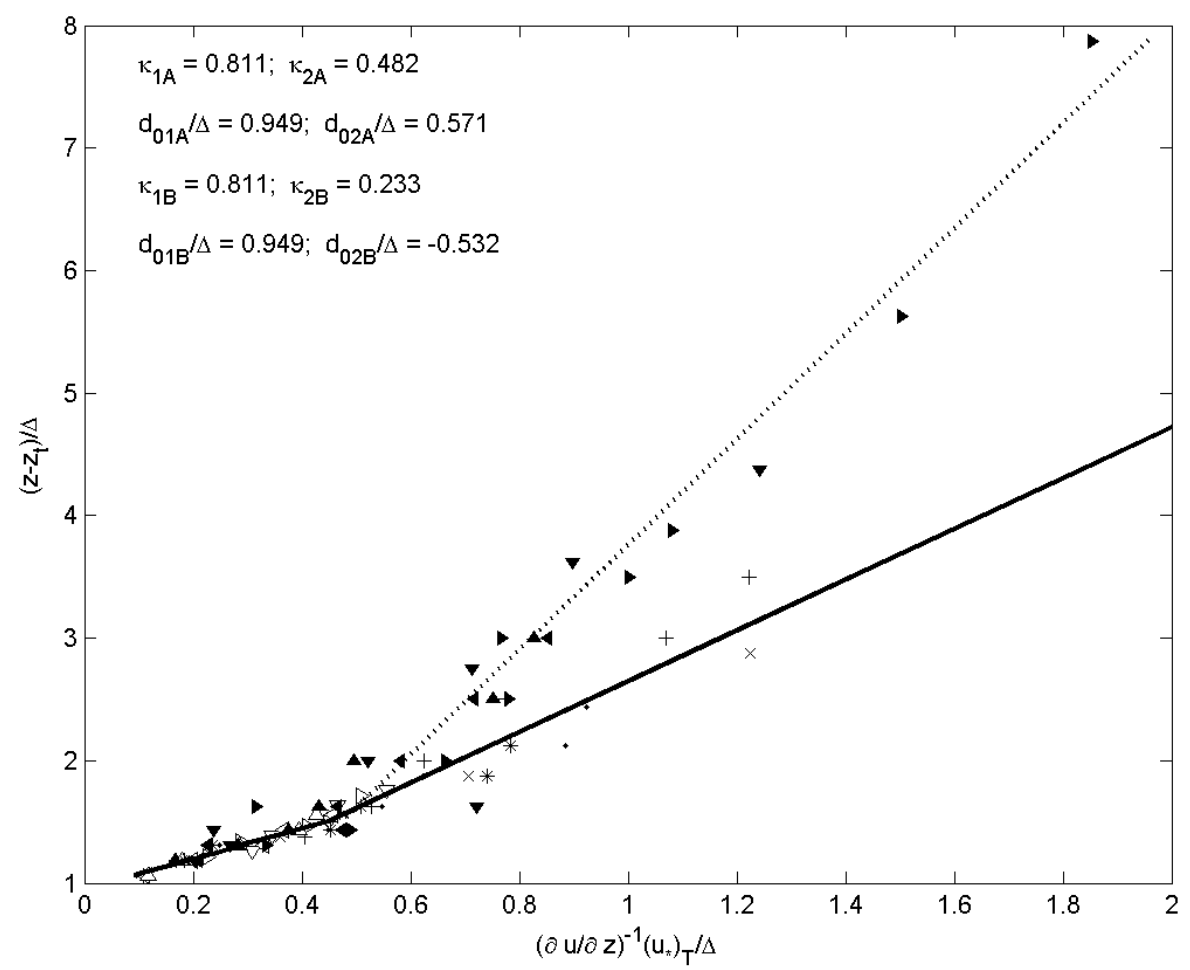

Fig. 5. Non-dimensional double-averaged inverse velocity shear plotted versus nondimensional elevation above the trough. The dotted line is a linear fit to the data from $\lambda / \Delta=10$ runs; the solid line is a linear fit to data from $\lambda / \Delta \approx 20$ runs. Just above the crest the fit is to the combined data (see Table 1 for symbol definitions).

locity there is considerable variability in the velocity profiles from run to run. This arises due to the complex inter-relationship between the bed geometry and the flow characteristics. The drag coefficient is not a simple function of the bed and flow parameters such as $h / \lambda, F r$ and $h / \Delta$. Also it is clear that both the power law and the segmented logarithmic profiles fit the data very well. Even though the power law yields a slightly better fit, the segmented nature of the data seems to be quite strong. Given that there is no clear evidence that the power law is better than the log law or vice versa, we will proceed by focusing on the logarithmic form of the velocity profile.

Because of the tendency of the velocity shear to increase rapidly as the boundary is approached, changes in the displacement thickness significantly change the character of the velocity profiles when plotted semi-logarithmically. In Fig. 7 the streamwise velocity from Run MNW 2 is plotted assuming that the bed origin is the trough, the mean bed elevation and the crest elevation. The profile that has the crest as the origin exhibits much more curvature than is present in the upper two curves. Also the shear velocity that characterizes the logarithmic fit to the lowest curve is less than half that from a fit to 


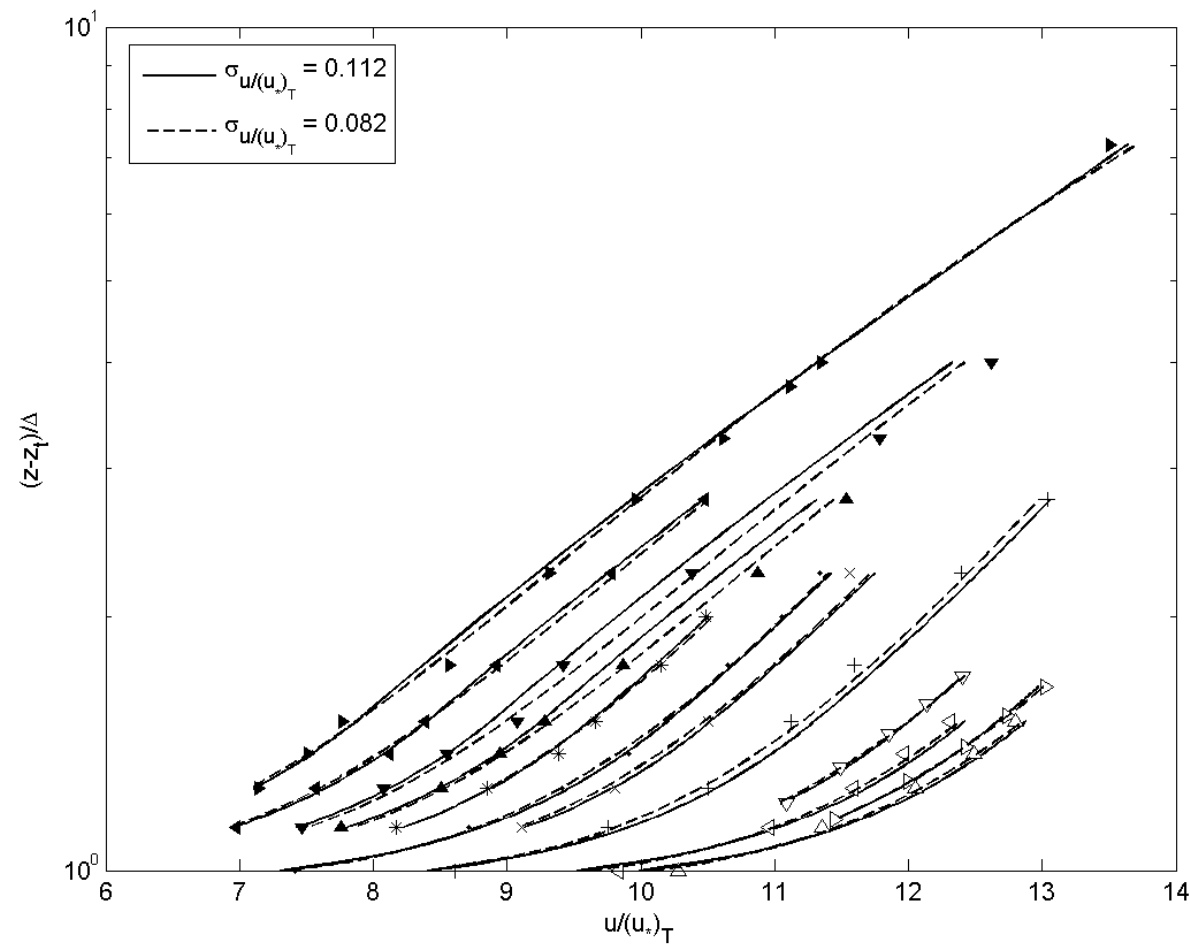

Fig. 6. Non-dimensional velocity profiles plotted semi-logarithmically with the origin at the trough of the dunes. The solid line is the segmented logarithmic profile, the dashed line is the power law velocity profile (see Table 1 for symbol definitions).

the uppermost profile. It should also be noted that the $R^{2}$ values for the upper two profiles are 0.999 and 0.998 both of which indicate they are highly logarithmic. Such small variability limits the sensitivity of determining the displacement thickness using the correlation coefficient. Consequently the inverse shear provides a much more sensitive means of determining the appropriate displacement thickness.

The S\&M approach assumed segmented velocity profiles implying a single displacement thickness (i.e., the origin was fixed at the local bed elevation). This implies a discontinuity in the velocity shear. In Fig. 5 the double-averaging approach exhibits segmentation in the inverse shear, not the velocity, and thus demands different displacement thicknesses between the inner and outer segments and indicates a matching shear profile. This, however, complicates the structure of the velocity profile plotted in semi-logarithmic space. Only a single displacement thickness can be defined for a given plot.

Clearly the velocity profiles above the crest level will exhibit logarithmic-like shape over a range of elevation but will exhibit greater complexity over the full range as is shown in Fig. 6 (where the origin is assumed to be at the trough elevation). Purely logarithmic velocity profiles (e.g., over a flat boundary) can be defined: 


$$
u=\frac{u_{*}}{\kappa} \log \frac{z}{z_{0}}
$$

where $u_{*}$ is one parameter associated with the flow, but a second constant $z_{0}$, the roughness parameter, must be found to completely specify the velocity profile. Using only the data from the logarithmic layer, this would be done by extrapolating the data to the point where the velocity goes to zero. For the segmented form that was discussed above, the velocity above the crest level would be described as

$$
\frac{u}{u_{*}}=\frac{1}{\kappa_{1}} \log \frac{\left(z-d_{01}\right)}{z_{01}} \quad \text { for } \quad z_{l}<z \leq z_{m}
$$

and

$$
\frac{u}{u_{*}}=\frac{1}{\kappa_{2}} \log \frac{\left(z-d_{02}\right)}{z_{02}} \quad \text { for } \quad z>z_{m}
$$

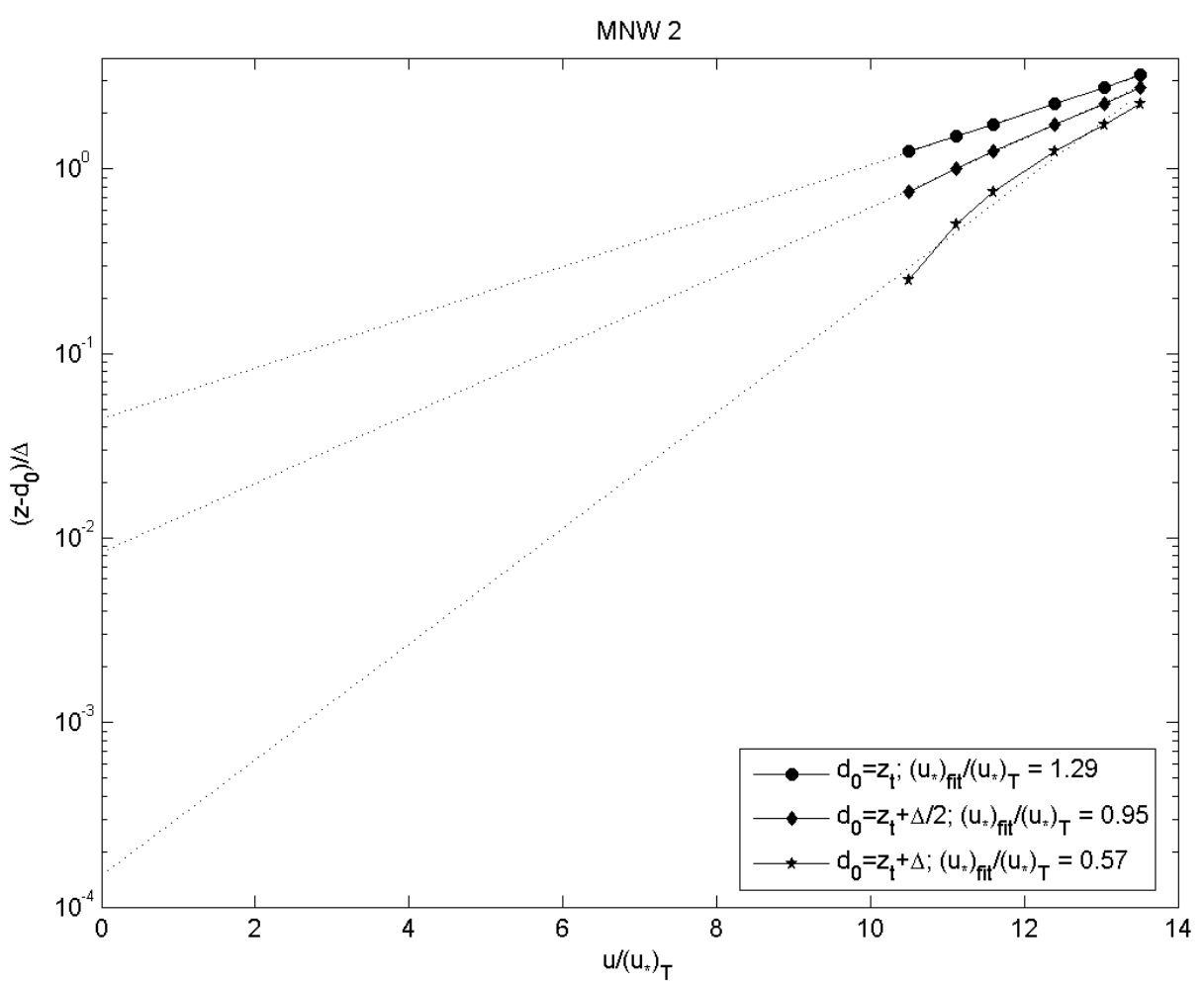

Fig. 7. Velocity profiles from Run MNW 2 assuming different values of displacement thickness (origin). Only data from $0.25 \Delta$ above the crest to $\sim 2 \Delta$ above the crest are included. The bottom curve assumes the origin is at the crest elevation, the origin of the middle curve is the mean elevation of the bed and the upper curve is plotted relative to the trough. 
where $\kappa_{1}$ and $\kappa_{2}$ reflect the different slopes in the inverse shear profiles and $z_{01}$ and $z_{02}$ are the elevations where the velocity extrapolates to zero. The matching point is found by matching the shear so that:

$$
z_{m}=\frac{\kappa_{1} d_{01}-\kappa_{2} d_{02}}{\kappa_{1}-\kappa_{2}} .
$$

Equation (7) suggests two straight line segments in the velocity profile plotted in semi-log space, but the differing displacement thicknesses $d_{01}$ and $d_{02}$ preclude that because the semi-log velocity plot can only have a single displacement thickness. Values for these variables in (7) are found from linear regressions to the inverse shear data and can be found in Table 2. (Note results for both single and intersecting line segments are included). The roughness parameter can be calculated by extrapolating a log fit to the lower part of the data and the roughness parameter for the outer segment can be found from matching the two equations in (7):

$$
\frac{z_{02}}{\Delta}=\frac{\left(z_{m}-d_{02}\right)}{\Delta} \exp \left[-\frac{\kappa_{2}}{\kappa_{1}} \log \left(\frac{z_{m}-d_{01}}{z_{01}}\right)\right] .
$$

However, this would ignore the data from below the crest elevation.

Table 2

Parameters describing the double-averaged velocity profiles.

$C_{1}, z_{1}, C_{2}, z_{2}$ are defined in (10)-(12), $\kappa_{1}, d_{01}, \kappa_{2}, d_{02}$ are defined in (7).

\begin{tabular}{|c|c|c|c|c|c|c|c|c|}
\hline $\begin{array}{l}\text { Data group } \\
\text { (runs) }\end{array}$ & $C_{1}$ & $z_{1} / \Delta$ & $C_{2}$ & $z_{2} / \Delta$ & $\kappa_{1}$ & $d_{01} / \Delta$ & $\kappa_{2}$ & $d_{02} / \Delta$ \\
\hline \multirow{2}{*}{ A (all) } & \multirow{2}{*}{-} & \multirow{2}{*}{-} & \multirow{2}{*}{9.42} & \multirow{2}{*}{0.210} & 0.285 & 0.086 & - & - \\
\hline & & & & & 0.811 & 0.949 & 0.233 & -0.709 \\
\hline \multirow{2}{*}{$\mathrm{B}(\lambda / \Delta \approx 20)$} & \multirow{2}{*}{-} & \multirow{2}{*}{-} & \multirow{2}{*}{10.22} & \multirow{2}{*}{0.140} & 0.556 & 0.777 & - & - \\
\hline & & & & & 0.821 & 0.949 & 0.482 & 0.571 \\
\hline \multirow{2}{*}{$\mathrm{C}(\lambda / \Delta \approx 10)$} & - & - & 8.84 & 0.378 & 0.258 & -0.064 & - & - \\
\hline & 7.573 & 0.381 & 10.39 & 0.463 & 0.810 & 0.957 & 0.233 & -0.532 \\
\hline \multirow{2}{*}{$\begin{array}{c}\mathrm{D}(\lambda / \Delta \approx 20 \\
\mathrm{LDV})\end{array}$} & \multirow{2}{*}{-} & \multirow{2}{*}{-} & \multirow{2}{*}{9.23} & \multirow{2}{*}{0.168} & 0.547 & 0.757 & - & - \\
\hline & & & & & 0.928 & 0.985 & 0.435 & 0.363 \\
\hline \multirow{2}{*}{$\begin{array}{c}\mathrm{E}(\lambda / \Delta \approx 20 \\
\mathrm{ADV})\end{array}$} & \multirow{2}{*}{-} & \multirow{2}{*}{-} & \multirow{2}{*}{11.35} & \multirow{2}{*}{0.126} & 0.615 & 0.843 & - & - \\
\hline & & & & & 0.725 & 0.904 & $*$ & $*$ \\
\hline
\end{tabular}

Bold numbers are used in producing Figs. 12, 14 and 15

${ }^{*}$ Group E runs contain insufficient data points in the outer part of the flow. 


\section{Averaged velocity below crest level}

In Fig. 8 the near-bed non-dimensional inverse shear has been added to the data that were plotted in Figs. 4 and 5. It is plain to see that the nature of the inverse shear profile changes markedly below the crest elevation, the non-dimensional inverse shear becoming very nearly constant at a value of approximately 0.1 . This indicates that the double averaged velocity varies nearly linearly in this region. In Fig. 9a the region below the crest elevation is expanded and only data from the $\lambda / \Delta \approx 20$ runs are included. In this expanded form it is apparent that the profile has a subtle reverse S-shape curvature rather than being constant. In Fig. $9 \mathrm{~b}$ only data from the $\lambda / \Delta=10$ runs appear and here the structure differs from that of the longer dunes, exhibiting an abrupt decrease in the inverse shear with distance above trough at about $70 \%$ of the dune height. The effect of this change in the shear is to produce a curving velocity profile (shear decreasing as the bed is approached) as seen in Fig. 9c. This is in keeping with the observation that velocity profiles over square-cross-section ribs oriented transverse to the flow transitioned from logarithmic to linear to exponential as the spacing decreased (Coleman et al. 2007). Here the decreasing aspect ratio is analogous to closer rib spacing. Nevertheless both of the structures in Figs. 9a and b are very subtle in comparison to the shear that exists above the crest level and assuming constant shear would not introduce significant error.

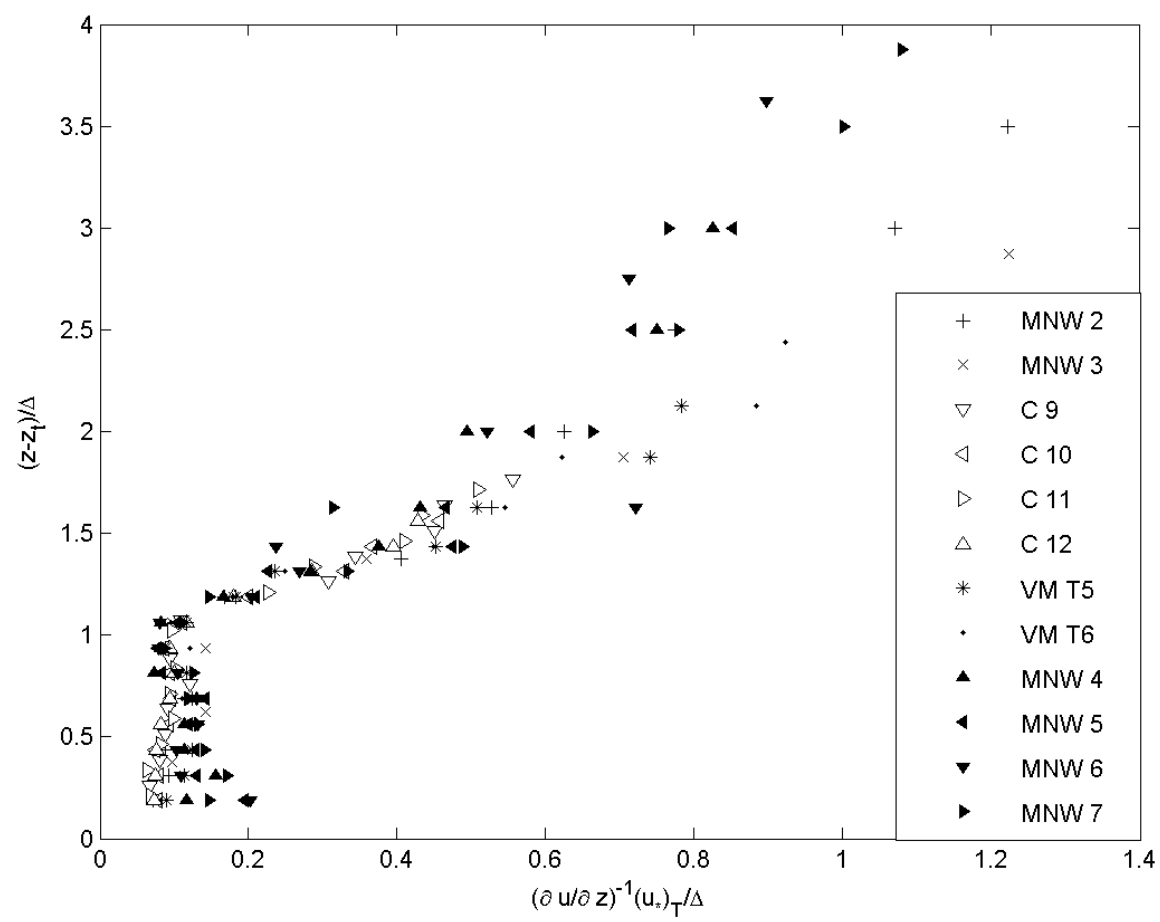

Fig. 8. Plot of non-dimensional inverse shear versus distance above the trough including near-bed data. 

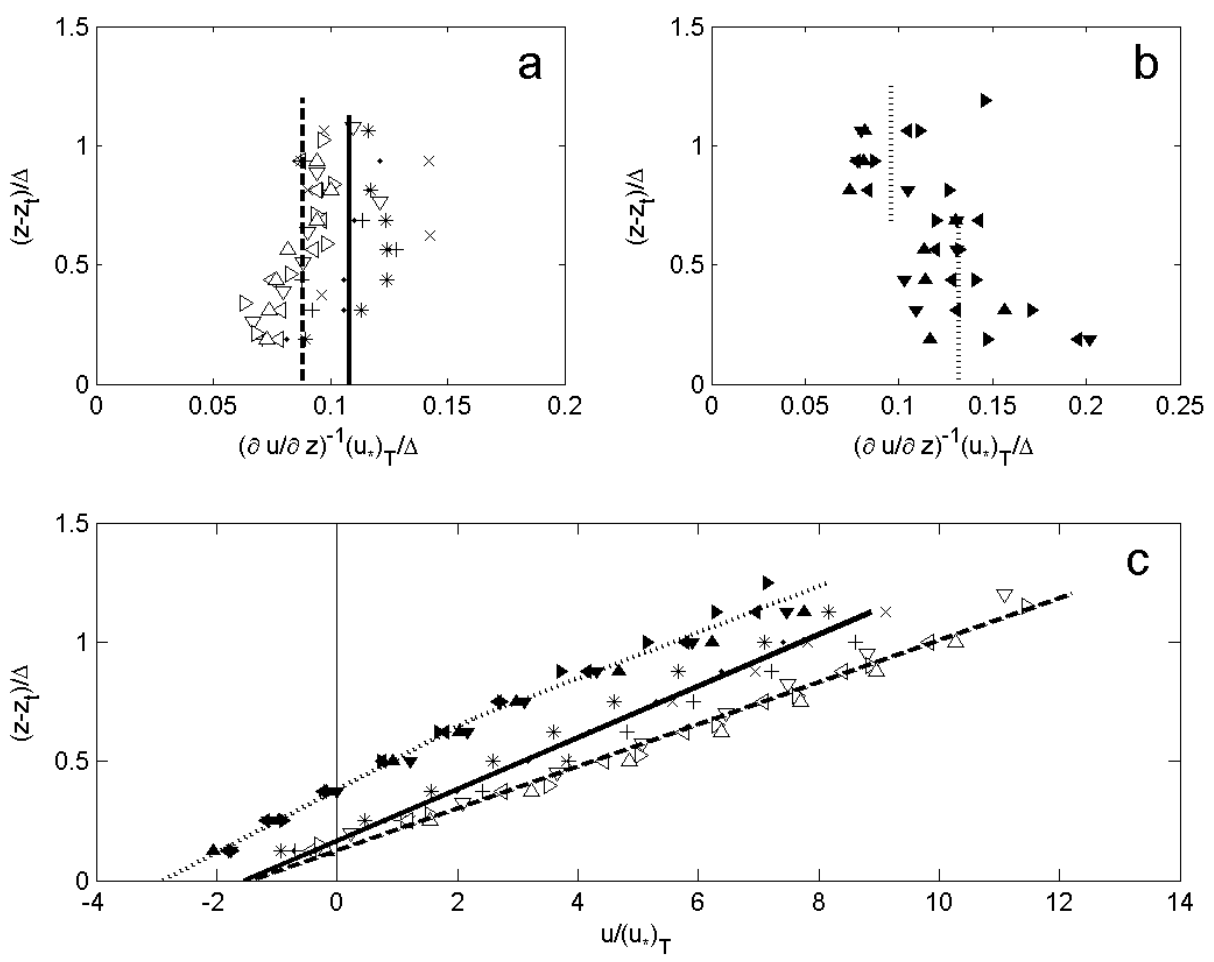

Fig. 9. Plots of non-dimensional inverse shear for $\lambda / \Delta \approx 20$ runs (a) and for $\lambda / \Delta=10$ runs (b); non-dimensional velocity for all runs (c). See Table 1 for symbol definitions.

In Fig. 9a it is also apparent that the data from McLean et al. (1994) and those from Coleman et al. (2006) exhibit the same curvature but are somewhat displaced from one another. The McLean et al. (1994) data were collected using a laser-Doppler velocimeter (LDV), the Coleman et al. (2006) data were collected using a laboratory acoustic-Doppler velocimeter (ADV). ADV measurements collected in the same flume and for the same flow conditions as MNW 2 (which were collected using an LDV) showed the nearbed velocity in the separation zone to be characterized by a velocity 'offset' in the positive streamwise direction relative to LDV measurements. That is, in the recirculation zone the upstream, reverse flow was not as strong in the ADV measurements as in the LDV measurements and the estimated location of the reattachment point was further upstream for the ADV measurements than for the LDV measurements. This is consistent with the non-dimensional velocity profiles plotted in Fig. 9c where the near-bed velocities from the Coleman (ADV) data are greater than the LDV measurements. Near the bed further downstream from reattachment and everywhere in the outer flow region the ADV and LDV measurements otherwise agree quite well. 


\section{Combined velocity profiles}

The velocity profiles in Fig. 9c can be approximated by

$$
\frac{u}{u_{*}}=C_{2} \frac{\left(z-z_{2}\right)}{\Delta},
$$

where $C_{2} \approx 10$ is a constant and $z_{2}$ is the level where the velocity is zero. For these separating flows $z_{2}$ will be above the trough level. For the $\lambda / \Delta=10$ runs (see Fig. 9b) one could argue that rather than this simple form one should divide this region so that:

$$
\frac{u}{u_{*}}=C_{1} \frac{\left(z-z_{1}\right)}{\Delta} \text { for } \frac{z}{\Delta} \leq 0.7
$$

and

$$
\frac{u}{u_{*}}=C_{2} \frac{\left(z-z_{2}\right)}{\Delta} \quad \text { for } \quad 0.7<\frac{z}{\Delta} \leq z_{l}
$$

where

$$
z_{l}=\frac{\Delta}{\kappa_{1} C_{2}}+d_{01}
$$

is found by using (7a) and matching the shear at that elevation. Furthermore, in either case we see that this formulation will provide a method for specifying $z_{01}$ without using a least squares fit to the data. By matching the velocity at $z_{l}$ we find

$$
z_{01}=\left(z_{l}-d_{01}\right) \exp \left[-\kappa_{1} C_{2} \frac{\left(z_{l}-z_{2}\right)}{\Delta}\right] .
$$

In Fig. 9c if the velocity profiles were extrapolated to the trough elevation, the $\lambda / \Delta=10$ runs would be significantly more negative than the $\lambda / \Delta \approx 20$ runs. This is because the separation zone for the $\lambda / \Delta=10$ runs encompasses a much greater portion of the sub-crest flow and hence leads to more negative double-averaged velocities near the bed.

Using eqs. (7) and (11) along with (8), (9), (12) and (13), and the parameters given in Table 2 that describe the various groups of runs, it is possible to generate smooth curves that estimate the various velocity profiles to within our knowledge of the shear velocity associated with the total shear stress $\left(u_{*}\right)_{T}$. Thus the total shear stress can be estimated by a least-squares fit of (7) and (11) to the measured double-averaged velocities with the shear velocity as a free constant. Figure 10 shows the results of such fits along with the ratios of the shear velocities from the fits to those that were measured. Clearly the fits are quite good, capturing most of the subtleties of the profiles. The overall standard deviation of the measured velocity about the fit is $0.24\left(u_{*}\right)_{T}$, about one quarter of the shear velocity associated with the 


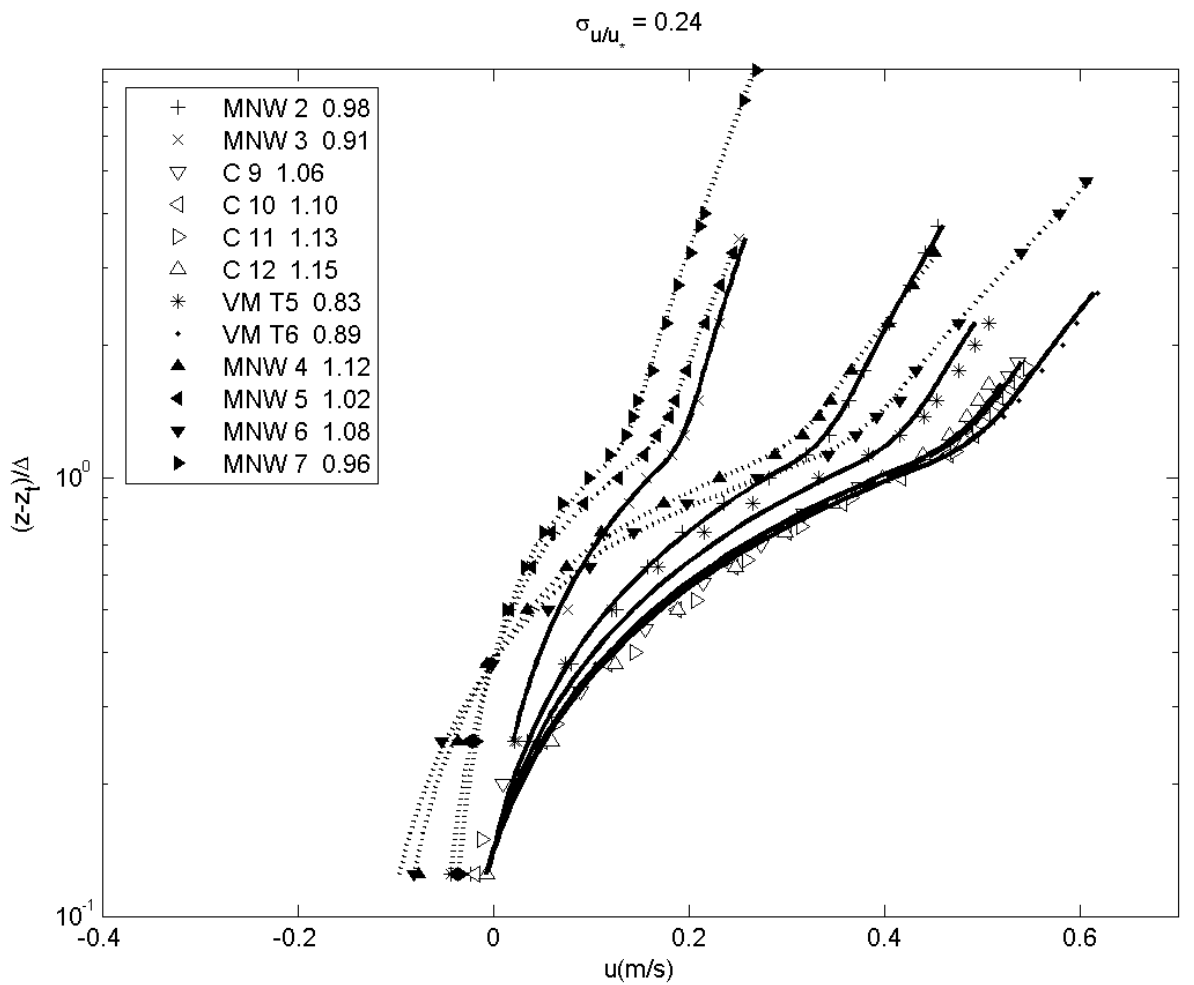

Fig. 10. Measured (symbols) and estimated profiles (bold lines) fitting (7) and (11) with parameters from Groups $\mathrm{B}$ and $\mathrm{C}$ in Table 2 to the measured velocities. The $\lambda / \Delta \approx 20$ runs are solid; the $\lambda / \Delta=10$ runs are dashed. The numbers to the right of the legend are the ratio of the shear velocity from the fit of (7) and (11) to the dimensional data to the measured shear velocity.

total stress. The mean of the ratio of the shear velocity is 1.02 and the standard deviation is 0.11 . The parameters that were used for these fits correspond to the numbers given for Groups $\mathrm{B}$ and $\mathrm{C}$ in Table 2 with segmented profiles in the log region for both groups and a double, matched linear profile for Group C. Further differentiating between the data collected using the LDV and that from the ADV (i.e., using the parameters from Groups D and $\mathrm{E}$ for the linear part of Group B reduces the overall standard deviation to $0.2\left(u_{*}\right)_{T}$.

Clearly such fits have many free constants and thus it is not at all surprising that the fits are good. Therefore it is of interest to see how good the fits are with fewer degrees of freedom. Assuming a single linear layer near the bed and a single log profile (Group A from Table 2) and normalizing by the shear velocity yields the results shown in Fig. 11. Clearly this is a much worse fit as the standard deviation of the velocity is 0.82 times the shear velocity. 


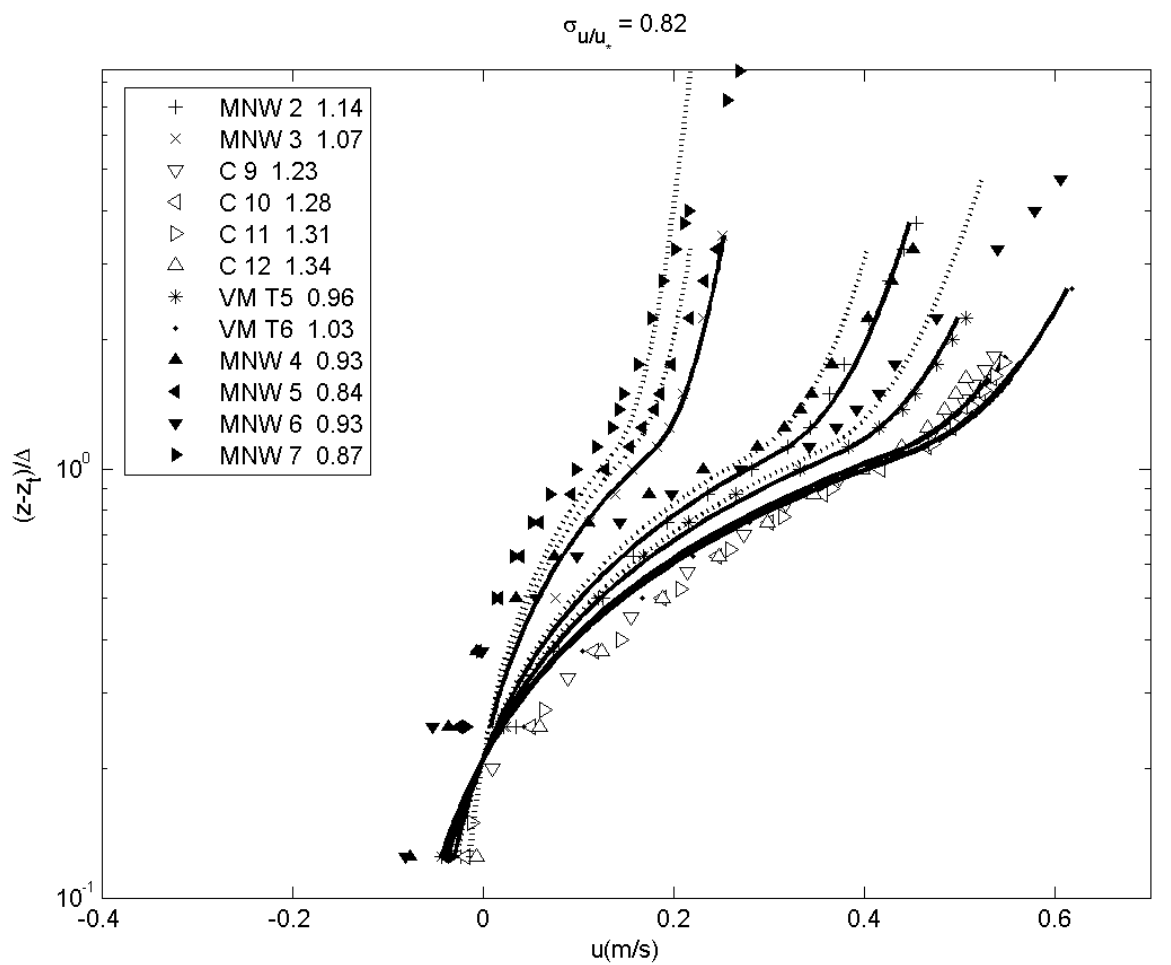

Fig. 11. Measured (symbols) and estimated profiles (bold lines). Here only four parameters from the first row in Table 2 are used for all the runs. The standard deviation of the normalized velocity is now $0.82\left(u_{*}\right)_{T}$.

Much of the error arises from trying to fit all the data near the bed even though there is a large discrepancy in the double-averaged velocity profiles near the bed for these two groups of runs.

In Table 2 it is apparent that the values for the intercept $z_{2} / \Delta$ for the Group B runs is about 0.14 whereas that for the Group C data is about 0.38. Wilbers (2004) suggested that the level of zero velocity in the separation zone over dunes dips down from the crest at an angle of approximately $\beta=10^{\circ}$ relative to the horizontal. Assuming the dune shapes are approximately triangular and extrapolating this line from the crest till it intercepts the stoss slope, one finds the reattachment point

$$
x_{r}=\frac{\lambda \Delta}{\Delta+(\lambda-\Delta / \tan \alpha) \tan \beta},
$$

where $\alpha$ is the angle of the lee face of the dune and $x_{r}$ is measured relative to the crest. Using this information a simple estimate for the intercept of the linear part of the velocity is 


$$
\frac{z_{2}}{\Delta}=1.44 \frac{x_{r}}{\lambda}-0.208
$$

Incorporating this simple expression for $z_{2}$ along with the other Group A values in Table 2, the standard deviation of the estimates is reduced to $0.48\left(u_{*}\right)_{T}$. By recognizing that the outer part of the flow is best described by a double log profile the standard deviation is reduced to $0.33\left(u_{*}\right)_{T}$.

Figures 10 and 11 arise by applying a fit of (7) and (11) to velocity profiles. It is of interest to know what kind of error might be associated with estimating the double-averaged velocity from an estimate of the total shear stress, by, for example, using the depth-slope product for a river channel. Figure 12 shows the results where the variation in $z_{2}$ according to (14) has been included. Here the non-dimensional velocity deviation is just about equal to the shear velocity. If the variation in $z_{2}$ is ignored the standard deviation increases to 1.58 times the shear velocity.

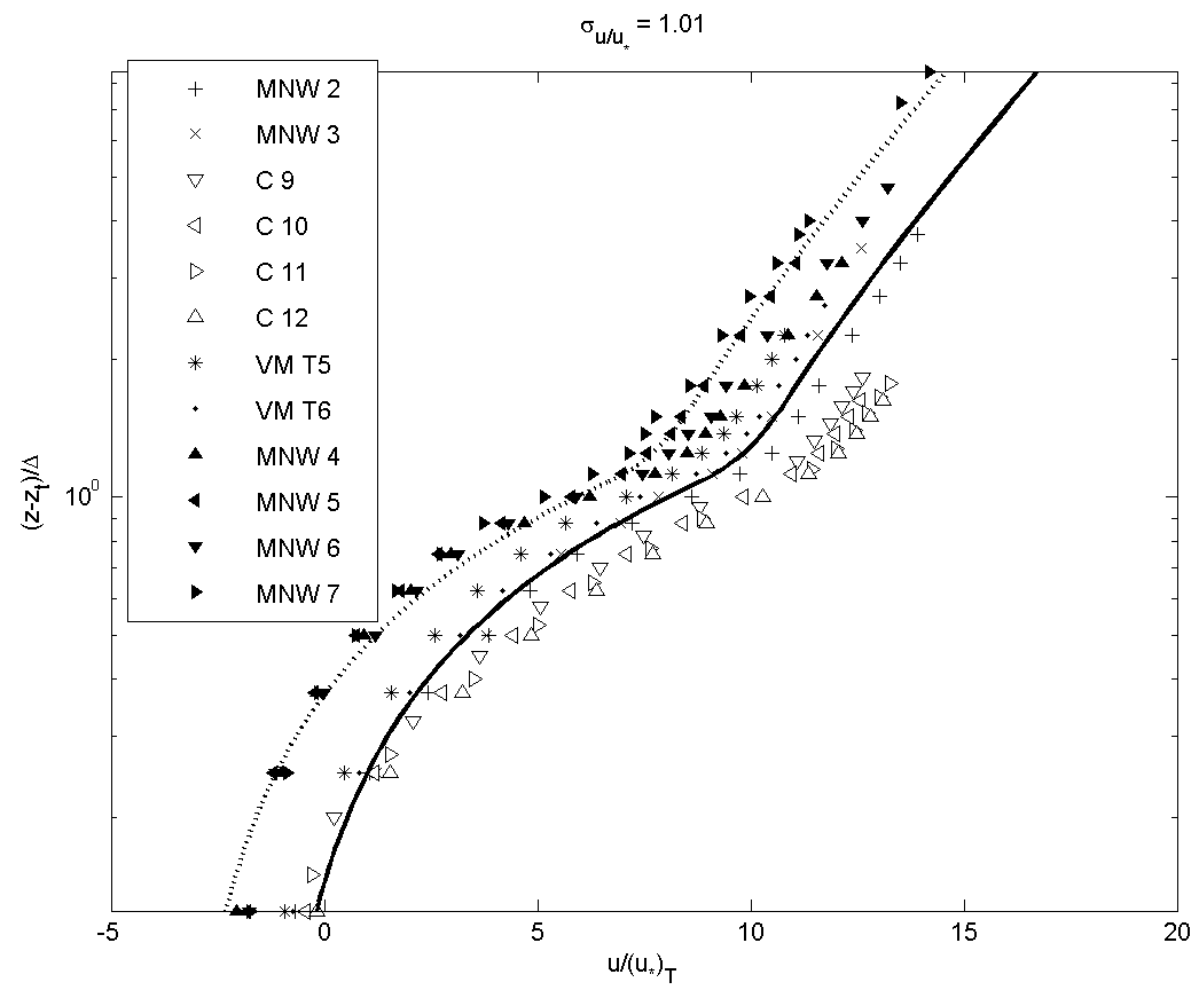

Fig. 12. Non-dimensionalized measured (symbols) and estimated velocity profiles (bold lines) using (14) to estimate the intercept $z_{2}$ of the linear velocity profiles, differentiating between dunes of different aspect ratios and assuming a double log profile in the outer part of the flow (bold numbers in Table 2). The standard deviation of the velocity is $1.01\left(u_{*}\right)_{T}$. 


\section{IMPLICATIONS FOR MIXING}

It has been common practice to utilize eddy viscosity to relate turbulent flux of momentum in turbulent boundary layers. Thus it is of interest to investigate how double-averaged velocity and stress fields in a very rough boundary layer might be related as suggested by McLean et al. (2007). The eddy viscosity can be assumed to be:

$$
K_{t}=-\left\langle\overline{u^{\prime} w^{\prime}}\right\rangle / \frac{\partial u}{\partial z} .
$$

In a uniform, flat boundary layer, the Reynolds stress increases linearly from zero at the free surface to a maximum near the bed then decreases back toward zero at the bed. The shear stress is commonly assumed to be constant within the log layer giving rise to a linearly increasing eddy viscosity throughout the log layer. As seen in Fig. 8 the inverse shear does not vary linearly, rather is nearly constant near the bed, then somewhat linear in the outer flow. Furthermore, as seen in Fig. 13a, over such rough topography the double-averaged Reynolds stress also is far from constant. At about the elevation of the crest the double averaged Reynolds stress is approximately equal to the total boundary shear stress, but below the crest (beginning even slightly above) the turbulent stress increases rapidly to about $50 \%$ greater than the total stress at approximately $0.7 \Delta$ then it decreases rapidly toward zero at the trough elevation. Combining this stress information with the inverse shear information using (15) we find the eddy viscosity profile shown in Fig. 13b. Clearly this eddy viscosity has a much more complex shape than the linear profile that is typically assumed in uniform boundary layers.

The eddy viscosity varies somewhat linearly from the trough elevation, and because the Reynolds stress also varies similarly just above the trough, the linear velocity profile results. If one assumes that $K_{t}=\kappa_{n b}\left(u_{*}\right)_{T}\left(z-z_{t}\right)$ then $\kappa_{n b}=0.26$ in this near bed region. However the rapid decline in the turbulent stress above $0.7 \Delta$ produces a decrease in the eddy viscosity until at about the crest elevation where it begins once again to increase somewhat linearly, but more rapidly than it does near the trough. Here if we assume that $K_{t}=\kappa_{\text {out }}\left(u_{*}\right)_{T}\left(z-z_{\text {out }}\right)$ then $\kappa_{\text {out }}=0.50$ and $z_{\text {out }}=0.85 \Delta$. This eddy viscosity (and presumably diffusivity; see, McLean et al. 2007) shape clearly has ramifications when trying to estimate the vertical flux of momentum (and mass and pollutants) from very rough boundaries. There is a considerable 'defect' in the mixing indicated by Fig. $13 \mathrm{~b}$ and what would be the case if one assumed a simple linear profile whose origin is at the trough. On the other hand assuming a linear profile whose origin was somewhat below the crest (at $0.85 \Delta$ above the trough) with an effective von Karman's constant of 0.50 would probably well-describe the mixing above the crest elevation. For the sub-crest region, the low effective von Karman's constant (0.26) suggests that either the turbulence is somewhat less than one would expect over a uni- 


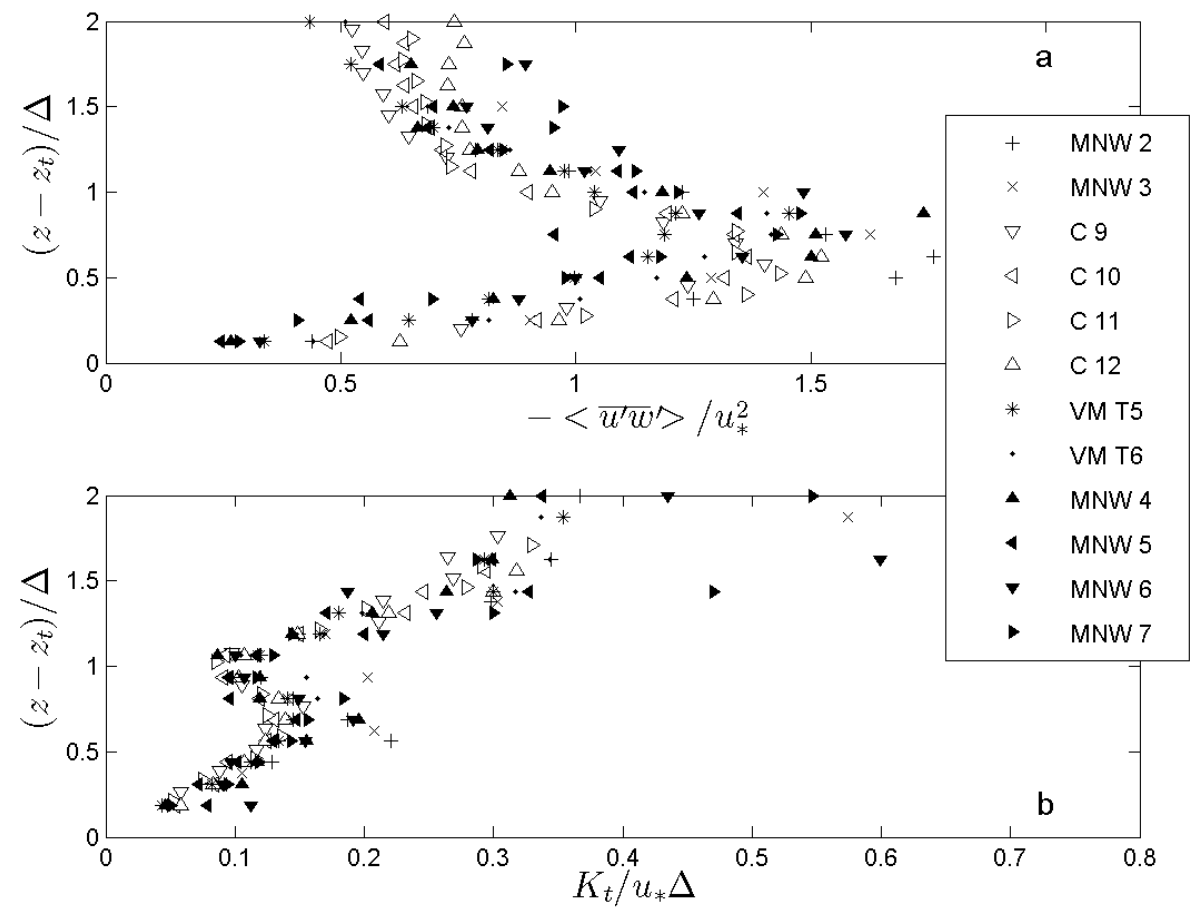

Fig. 13. Non-dimensional double-averaged: (a) Reynolds shear stress and (b) eddy viscosity profiles.

form boundary or the mixing length is somehow more limited. Given the very high turbulence levels that are measured in the separation region, it is doubtful that the turbulent velocity scale is somehow smaller over these rough beds, therefore the smaller effective von Karman's constant is likely tied to a limiting of the size of the turbulent eddies.

\section{OTHER LABORATORY PROFILES}

Herein we have so far limited the discussion to highly two-dimensional laboratory studies conducted over fixed beds. These do not, however, reflect the character of many natural flows. Therefore it is of interest to compare measurements from other types of flows to these. For example, Best and Kostaschuk (2002) conducted similar measurements over fixed dune shapes that were modeled after naturally occurring dunes in the Fraser River of British Columbia. In nature these dunes differ from the dune shapes investigated above in that they are 'low-angle dunes' wherein they are longer and lower, with a lee slope that is much less than the angle of repose of sand. These bedforms are found to be in the transition from dunes to the upper regime plane bed, with increasing lag between sediment transport and the topography (due to increasing suspended sediment load) leading to gradual decay in the dunes. The low slope of the bed in the lee of the crests of these bedforms 
means that the flow separates only occasionally, therefore the double averaged flow is everywhere downstream even near the trough. Best and Kostaschuk (2002) created scaled two-dimensional dune shapes in the laboratory that had the same low-angle profile of the natural Fraser dunes.

Maddux et al. (2003a, b) present detailed measurements of the flow over three-dimensional perturbations of the dune shapes investigated in Runs MNW 2 and MNW 3 (see Table 1) with approximately the same flow conditions. The shape was perturbed by maintaining troughs that were of constant elevation and ran straight across the flume, whereas the crests varied in height by $50 \%$ of the mean $(0.04 \mathrm{~m})$ with a $30^{\circ}$ lee slope everywhere. This geometry produces a crestline that not only varied in height but also meandered across the flume with the largest crest heights upstream of the lowest crest heights. Thus wavelengths varied with position across the flume. The perturbed shapes were alternated such that high crests were followed in the streamwise direction by low crests.

These additional sets of measurements provide three example runs of flows that differ significantly from the 'pristine' 2-d flows discussed thus far. Their characteristics are included at the bottom of Table 1. In Fig. 14 the non-dimensional velocities for these runs are plotted along with the MNW 2,

$\sigma_{\mathrm{u} / \mathrm{u}}=1.95$

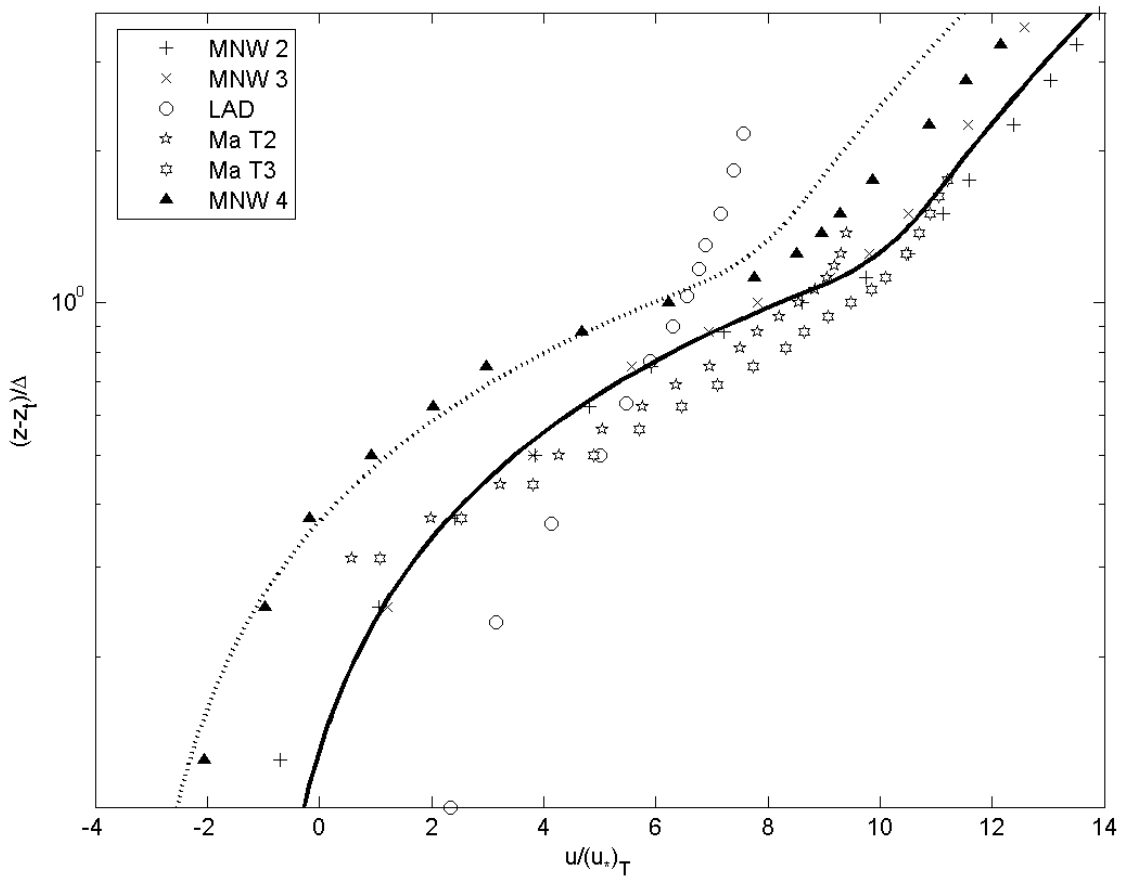

Fig. 14. Non-dimensional double-averaged velocity profiles from two-dimensional low-angle dunes (LAD) and three-dimensional dune shapes (Ma T2 and T3). Also included are MNW 2, 3 and 4 for reference. The bold lines are the estimated velocity profiles as in Fig. 12. 


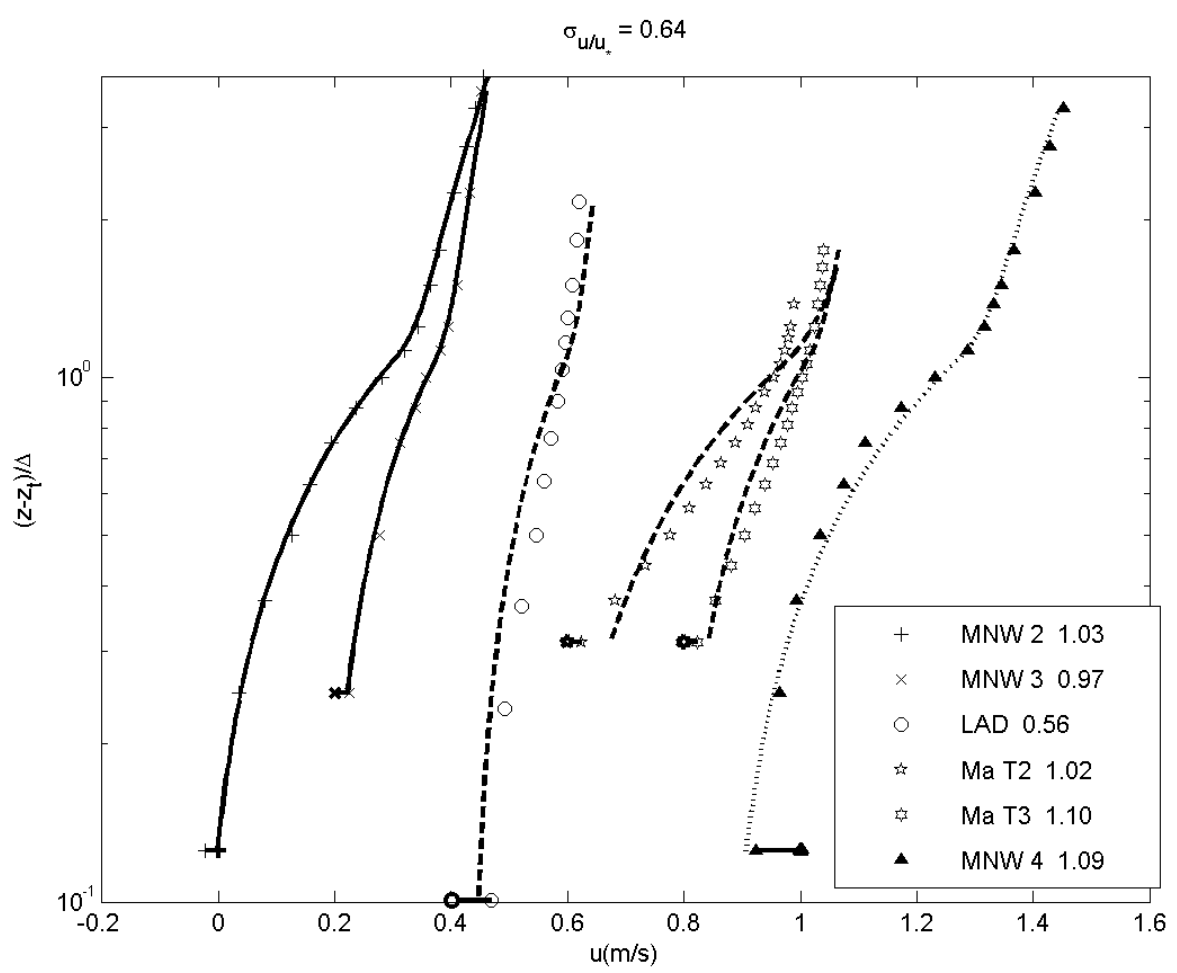

Fig. 15. Velocity profiles using fits of (7) and (11) (and bold parameters from Table 2) fit to double-averaged velocity measurements. The bold symbols (connected by bold lines to the bottom data points) indicate zero spatially averaged velocity.

MNW 3 and MNW 4 runs as comparison. In this plot the shear velocity associated with the measured total shear stress is used in conjunction with (7) and (11). Not surprisingly the low-angle dune measurements are significantly larger near the bed because of the absence of a continual separation zone. All three of these runs exhibit significantly less structure than the other runs. Again one would expect less structure in the low-angle dune case where the mixing associated with separation is for the most part missing. In the threedimensional flows the variation in dune height apparently smears the shear in the velocity over a larger vertical range. Figure 15 presents the results of simply fitting (7) and (11) to the velocity profiles, with the ratio of the fitted shear velocity to the measured shear velocity shown in the legend. The lowangle dune (LAD) case clearly indicates that the 'measured' total shear stress is perhaps too large. The value of $z_{2}$ for the LAD velocity profile was found using (14) and assuming the reattachment position $x_{r}=0$.

It is of interest to investigate the inverse shear and the velocity in the near bed region (sub-crest elevations). In Fig. 16a the inverse shear is shown and the LAD data tends to show that the innermost part of the velocity profile is approximately linear only in the lowest half of the sub-crest region. At 

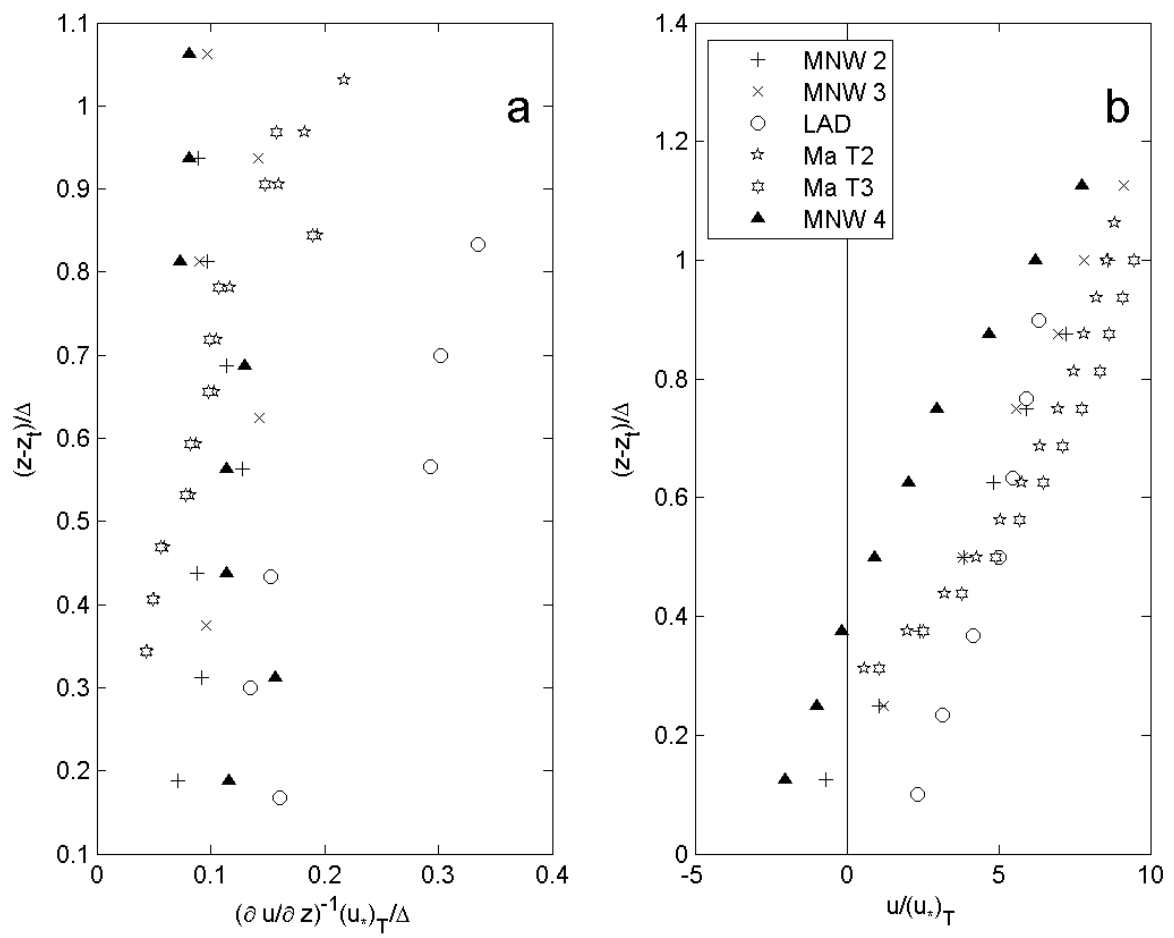

Fig. 16. Non-dimensional inverse shear (a) and non-dimensional velocity profiles (b) for the two-dimensional low-angle dunes (LAD) and three-dimensional dune shapes (Ma T2 and T3). Also included are MNW 2, 3 and 4 for reference.

about $0.5 \Delta$ above the trough elevation the inverse shear makes a rapid transition to a region of weak, somewhat linear, variation. On the other hand we see with the three-dimensional bedforms that there is no place where the inverse shear is constant; even in the near-bed region the inverse shear grows linearly. This leads to the more concave downward shape of the velocity profile for both of the runs (see Fig. 16b).

In Fig. 17a the non-dimensional Reynolds stress is shown for these cases. As is discussed by Maddux et al. $(2003 \mathrm{a}, \mathrm{b})$ the Reynolds stress for the three-dimensional cases is markedly less than one would expect from the two-dimensional measurements. This is due to secondary circulation that was not present in the 2-d cases. The low-angle dunes also exhibit even smaller non-dimensional stress. This could be partly due to an overestimated total stress (as mentioned above), but it is also likely because the flow only separates on occasion and separation is the main source of turbulence. Furthermore when the flow separates it only does so on a small portion of the lee slope, toward the trough. Consequently the maximum in the shear stress occurs around $0.2 \Delta$ instead of around $0.7 \Delta$ for the $2-\mathrm{d}$ cases.

Finally, in Fig. 17b the eddy viscosities for these three cases are very different from the 2-d cases, for example, none exhibit the sharp dip just below 


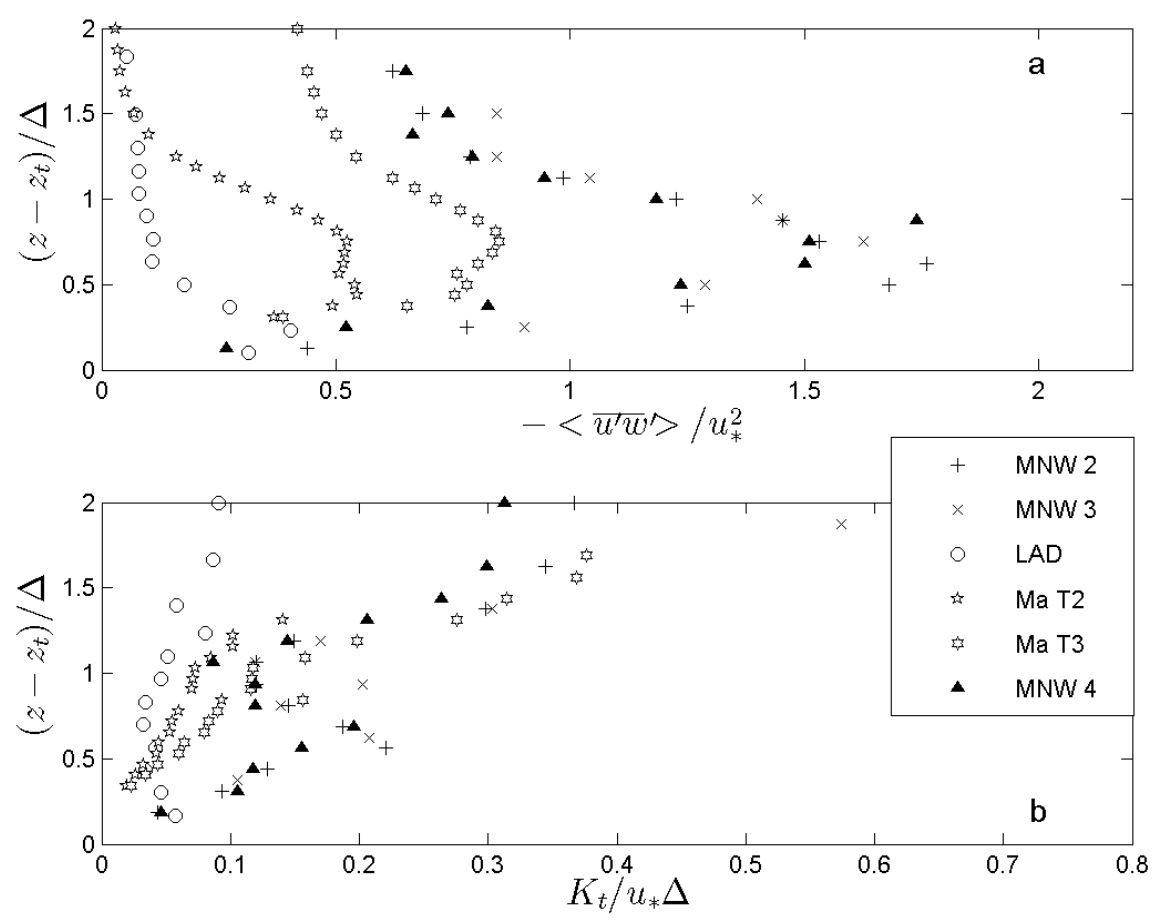

Fig. 17. Non-dimensional double-averaged Reynolds shear stress (a) and eddy viscosity profiles (b) for the two-dimensional low-angle dunes (LAD) and threedimensional dune shapes (Ma T2 and T3). Also included are MNW 2, 3 and 4 for reference.

the crest elevation. The dip arose due to a combination of rapid decrease in the Reynolds stress with distance from the bed under conditions of constant shear. In the low-angle dune case, near the trough, the eddy viscosity actually decreases with distance from the bed because the maximum stress is so near the trough elevation. Further from the bed the inverse shear begins varying linearly where the stress decrease is relatively small so the inverse shear increase is greater than the decrease in stress. For the 3 -d cases the inverse shear is also linearly increasing over the sub-crest region, the maximum stress occurs further from the bed and the variation in the bedform height smears the decrease in the stress over a wider portion of the profile, apparently enough that the linear increase in the inverse shear dominates over the decrease in stress.

\section{CONCLUSIONS}

Spatial averaging can be useful in characterizing the flow over very rough boundaries; however, great care must be taken in the process. Some methods of averaging such as that undertaken by S\&M may reduce the degrees of 
freedom and provide a basis for empirical expressions that predict quantities like the form drag or total stress fairly accurately, but may fail to predict the skin friction because the averaging technique inadvertently mixes processes and degrades the applicability of the assumptions. Double averaging over horizontal planes or thin slab volumes, on the other hand, allows the incorporation of conservation of mass and momentum equations that aid in the interpretation of the results.

- Smith-McLean average velocity profiles and Cartesian average profiles differ dramatically because of the fundamental difference in the nature of the average, the former omitting the separation region for example. The S-M method predicts form drag fairly well but is not able to predict the skin friction reliably.

- Above the crest elevation the inverse shear is characterized by a concave upward shape that could be interpreted as signifying a power law velocity variation. However, it also appears to be well-described by two linear segments that intersect at about 1.5 bedform heights above the crest. The latter description leads to matching logarithmic profiles; however, if the shear is matched, semi-logarithmic plots of velocity will not yield two straight line segments because the displacement thicknesses for the two segments are different.

- The steep dune shapes and the longer, lower shapes exhibit the same slope in the inverse shear just above the crest, in the lower log segment, but differ significantly in the outermost segment.

- Below the crest elevation the inverse shear abruptly changes its nature to be nearly constant, indicating linear velocity variation with distance from the bed.

a There are subtle differences in the inverse shear between the dunes of different mean slope but there is little loss in accuracy to assume that velocity shear is $\sim 10\left(u_{*}\right)_{T} / \Delta$ near the bed.

- The greatest difference in the near bed velocity between the dunes of different aspect ratio has to do with the velocity intercept (the velocity interpolated to the trough elevation). The short dunes have more negative intercept velocities because the separation zone encompasses more of the sub-crest region.

- Double average velocity estimates for flow over two-dimensional bedforms agree to within a standard deviation of about $\left(u_{*}\right)_{T} / 3$ if a fit to measured velocities is possible. The resulting shear velocity agrees to within a standard deviation of $\sim 11 \%$. If no velocity measurements are used, the standard deviation of the velocity estimates will be about $\left(u_{*}\right)_{T}$.

a Two-dimensional low-angle dune and three-dimensional dune shape measurements exhibit some of the same characteristics as the 2-d 
cases, but also some significant differences. The low-angle dunes, because separation occurs only intermittently, and over a small portion of the lee, exhibit constant shear only very near the trough and relatively low turbulence levels $\sigma^{2}$. The 3 -d cases exhibit no regions of constant shear and the gradients in the velocity and Reynolds stress are smeared out due to the variation in the bedform heights. It is likely that natural dunes would also exhibit this behaviour and have less structure to their double averaged velocity than was exhibited by the 2-d forms discussed here.

\section{References}

Barenblatt, G.I. (2003), Scaling, Cambridge University Press, Cambridge.

Best, J.L., and R.A. Kostaschuk (2002), An experimental study of turbulent flow over a low-angle dune, J. Geophys. Res. 107, 3135-3154.

Coleman, S.E., V.I. Nikora, S.R. McLean, T.M. Clunie, E. Schlicke, and B.W. Melville (2006), Equilibrium hydrodynamics concept for developing dunes, Phys. Fluids 18, 10, 105104-1-12.

Coleman, S.E., V.I. Nikora, S.R. McLean, and E. Schlicke (2007), Spatially-averaged turbulent flow over square ribs, J. Eng. Mech. ASCE 133, 194-204.

Finnigan, J.J. (2000), Turbulence in plant canopies, Ann. Rev. Fluid Mech. 32, 519571.

Gimenez-Curto, L.A., and M.A. Corniero Lera (1996), Oscillating turbulent flow over very rough surfaces, J. Geophys. Res. 101, C9, 20,745-20,758.

Maddux, T.B., J.M. Nelson, and S.R. McLean (2003a), Turbulent flow over three dimensional dunes: 1. Free surface and flow response, J. Geophys. Res. 108, F1, 10-1-20, DOI: 10.1029/2003JF000017.

Maddux, T.B., S.R. McLean, and J.M. Nelson (2003b), Turbulent flow over three dimensional dunes: 2. Fluid and bed stresses, J. Geophys. Res. 108, F1, 111-17, DOI: 10.1029/2003JF000018.

McLean, S., and V. Nikora (2006), Characteristics of turbulent unidirectional flow over rough-beds: Double-averaging perspective with particular focus on sand dunes and gravel beds, Water Resour. Res. 42, W10409.

McLean, S.R., J.M. Nelson, and S.R. Wolfe (1994), Turbulence structure over twodimensional bed forms: Implications for sediment transport, J. Geophys. Res. 99, C6, 12,729-12,747.

McLean, S.R., S.R. Wolfe, and J.M. Nelson (1999), Spatially averaged flow over a wavy boundary revisited, J. Geophys. Res. 104, C7, 15,743-15,753.

McLean, S.R., J.M. Nelson, and L. Gary (2007), Suspended sediment in the presence of dunes. In: C.M. Dohmen-Janssen and S.J.M.H. Hulscher (eds.), River, Coastal and Estuarine Morphodynamics (RCEM), Taylor \& Francis Group, Leiden, 611-618.

Nikora, V.I., and S.R. McLean (2001), Environmental flows over rough beds: a spatial averaging approach, Proc. 3rd Intern. Symp. on Environmental Hydraulics, Arizona, USA (CD-ROM). 
Nikora, V.I., D.G. Goring, I. McEwan, and G. Griffiths (2001), Spatially-averaged open-channel flow over a rough bed, J. Hydraul. Eng. ASCE. 127, 123-133.

Nikora, V.I., K. Koll, S. McLean, A. Dittrich, and J. Aberle (2002), Zero-plane displacement for rough-bed open-channel flows, Proc. Intern. Conf. on Fluvial Hydraulics River Flow 2002, Sept. 4-6, 2002, Louvain-la-Neuve, Belgium, 83-92.

Nikora, V., K. Koll, I. McEwan, S. McLean, and A. Dittrich (2004), Velocity distribution in the roughness layer of rough-bed flows, J. Hydraul. Eng. ASCE 130, 7, 1036-1042.

Nikora, V.I., I.K. McEwan, S.R. McLean, S.E. Coleman, D. Pokrajac, and R. Walters (2007a), Double-averaging concept for rough-bed open-channel and overland flows: Theoretical background, J. Hydraul. Eng. ASCE 133, 8, 873-883.

Nikora, V., S. McLean, S. Coleman, D. Pocrajac, I. McEwan, L. Campbell, J. Aberle, D. Clunie, and K. Koll (2007b), Double-averaging concept for rough-bed open-channel and overland flows: Applications, J. Hydraul. Eng. ASCE 133, 8, 884-895.

Raupach, M.R., R.A. Antonia, and S. Rajagopalan (1991), Rough-wall turbulent boundary layers, Appl. Mech. Rev. 44, 1, 1-25.

Smith, J.D., and S.R. McLean (1977), Spatially averaged flow over a wavy surface, J. Geophys. Res. 83, 12, 1735-1746.

van Mierlo, M.C.L.M., and J.C.C. de Ruiter (1988), Turbulence measurements above artificial dunes, Delft Hydraulics Laboratory, Report No. Q789.

Wilbers, A. (2004), The development and hydraulic roughness of subaqueous dunes, Ph.D. Dissertation, University of Utrecht.

Received 29 November 2007 Accepted 23 April 2008 